\title{
End-to-End Verifiable Elections in the Standard Model $^{\star}$
}

\author{
Aggelos Kiayias ${ }^{\dagger \ddagger}$, Thomas Zacharias ${ }^{\ddagger}$, and Bingsheng Zhang ${ }^{\ddagger}$ \\ Dept. of Informatics and Telecommunications, \\ National and Kapodistrian University of Athens, Greece \\ \{aggelos, thzacharias, bzhang\}@di.uoa.gr
}

\begin{abstract}
We present the cryptographic implementation of "DEMOS", a new e-voting system that is end-to-end verifiable in the standard model, i.e., without any additional "setup" assumption or access to a random oracle (RO). Previously known end-to-end verifiable e-voting systems required such additional assumptions (specifically, either the existence of a "randomness beacon" or were only shown secure in the RO model). In order to analyze our scheme, we also provide a modeling of end-toend verifiability as well as privacy and receipt-freeness that encompasses previous definitions in the form of two concise attack games.

Our scheme satisfies end-to-end verifiability information theoretically in the standard model and privacy/receipt-freeness under a computational assumption (subexponential Decisional Diffie Helman). In our construction, we utilize a number of techniques used for the first time in the context of e-voting schemes that include utilizing randomness from bitfixing sources, zero-knowledge proofs with imperfect verifier randomness and complexity leveraging.
\end{abstract}

\section{Introduction}

In an end-to-end (E2E) verifiable election system, voters have the ability to verify that their vote was properly cast, recorded and tallied into the election result. Intuitively, the security property that an E2E verifiable election intends to capture is the ability of the voters to detect a malicious election authority that tries to misrepresent the election outcome. E2E verifiability is a strong level of security for election systems that has been widely accepted as a fundamental requirement for their adoption, see e.g., [39].

In more details, E2E verifiability mandates that the voter can obtain a receipt at the end of the ballot casting procedure that can allow her to verify that her vote was (i) cast as intended, (ii) recorded as cast, and (iii) tallied as recorded. Furthermore, any external third party should be able to verify that the election

$\dagger$ Research was supported by ERC project CODAMODA.

$\ddagger$ Research was supported by project FINER, Greek Secretariat of Research and Technology funded under action "ARISTEIA 1."

* The authors are ordered alphabetically and the third author is the corresponding author. 
procedure is executed properly. In fact, it is imperative that the receipts in an E2E system are delegatable i.e., the voter may delegate the task of verifiability to any interested third party, for instance an international organization of the voters' choosing that aggregates the task of verification. This requirement, as well as the fact that it should be infeasible for the voter to use her receipt as a proof of the way she voted (this is necessary to deter vote-selling/buying), make the design of end-to-end verifiable systems a challenging problem.

All known e-voting systems that offer $\mathrm{E} 2 \mathrm{E}$ verifiability provide it under some setup assumption or in the random oracle (RO) model. Notably, E2E verifiability can be argued (note that it is never formally proven before) for Helios [1] in the RO model while for Remotegrity ${ }^{1}$ [44] in the model where a trusted party (a "randomness beacon") provides a stream of unbiased and unpredictable random coins. More general approaches for defining auditable multiparty computation (MPC) have recently been proposed [3] and also rely on a setup assumption such as a CRS.

A critical shortcoming of using setup assumptions for establishing E2E verifiability in e-voting is the fact that the voters will be required to make a "leap of faith" and accept the setup assumption in order to accept the election result. This can be an unfortunate state of affairs: since the election authority (EA) cannot unequivocally convince the voters that the election is correct, then the election outcome can be always subject to dispute.

Our Results. Motivated by the above, we design a new e-voting system that we can prove E2E verifiable information theoretically in the standard model, i.e., without any setup assumption except the existence of a bulletin board (BB) which provides a consistent view of the election transcript. Our result is further strengthened by the fact that we make the absolute minimal assumptions on the computation capabilities of the voters: voters are merely modeled as finite state transducers and thus are incapable of performing any cryptographic operation during ballot-casting (note the auditing stage after the election would require the capability of cryptographic operations but they can be performed at any time, in the post-election stage).

To accomodate the analysis of our system we provide a model for E2E verifiability and voter privacy/receipt-freeness. Our model for E2E verifiability is inspired from input-indistinguishable computation of Micali, Pass and Rosen [36] since in their setting they are also faced with proving security for multiparty computation in the standard model (note however they do not deal with E2E verifiability/auditability in their setting). In our modeling, the election system involves three types of entities, the voters $V_{1}, \ldots, V_{n}$, the election authority (EA), and the bulletin board (BB) whose only role is to provide storage for the election transcript for the purpose of verification. Voters submit their votes by engaging in the ballot casting protocol to the EA and they are not allowed to interact with each other. Our definition of end-to-end verifiability considers a

\footnotetext{
${ }^{1}$ Note that Remotegrity itself is only a "front-end" type of system. It will be E2E verifiable if combined with Scantegrity-II [14] as suggested by the authors of the paper.
} 
very powerful adversary that is computationally unbounded and completely controls the EA. On the other hand, BB is completely passive and is only writeable by the EA and readable by anyone. The definition is satisfied, if and only if the adversary is incapable of evading being detected when it manipulates the election result as long as a number of voters perform the verifiability procedure honestly. Voter privacy on the other hand, considers an adversary that has full access to all the the voters' receipts, views of the ballot casting protocol as well as it may control of a number of malicious voters. For any election tally, the adversary should be incapable of distinguishing the way honest voters vote.

Our construction cherry picks ideas put forth in previous works, specifically, code-voting and double ballots from $[12,13]$, but also introduces a number of novel elements that enable us to prove E2E verifiability in the standard model. In order to achieve verifiability, our system utilizes a novel ZK proof for candidate encoding correctness and collects coins from the voters to form the challenge (specifically, a single random coin per voter). Given that the majority of voters cannot be assumed to be properly following the protocol, the sequence of voter contributed randomness is a particularly "weak source" that cannot be used for arguing the integrity of the election in a direct way - as we argue it is a very weak source akin to adaptive bit-fixing sources [35]. We then show (i) how it is possible to perform our ZK proof with a verifier that has imperfect randomness (just a min-entropy source), (ii) how to produce a (sufficiently long) sequence of min-entropy challenge from the random bits contributed by the voters. The tools that are important in our construction include a generalization of the Schwartz-Zippel lemma $[42,45]$ for imperfect randomness and a suitable strategy for dividing the coins of the voters so that the entropy is not lost due to the adversarial strategy of the EA (who also controls a number of voters). Using these techniques we design a novel ZK protocol and we prove unconditionally end-to-end verifiability for our scheme. For voter privacy, we utilize complexity leveraging to construct a simulator that is capable of reducing a voter privacy attack to a subexponential DDH distinguisher and hence our system offers privacy and receipt-freeness under a computational assumption.

In summary, our e-voting system is the first construction achieving the properties E2E verifiability and voter privacy/receipt-freeness in the standard model. Furthermore, we prove E2E verifiability information theoretically assuming the voters are computationally restricted transducers that hence are incapable of performing any cryptographic operation during ballot casting. The only assumptions we make are subexponential Decisional Diffie Hellman assumption (for voter privacy/receipt-freeness) and a consistent bulletin board board. We remark that a consistent bulletin board can be easily seen to be a tight condition since without it, it is easy to verify that $\mathrm{E} 2 \mathrm{E}$ verifiability of the election cannot be achieved: by controlling the $\mathrm{BB}$, an adversarial EA can distribute voters to their own separate "islands" where within each one the voters will have their own verifiable view of an election result that can be - in reality - completely skewed. Implementing a consistent bulletin board is beyond our scope, however we note that it can be achieved in the standard model using Byzantine agrement 
(BA) (for BA, see e.g., [25]) by assuming secure channels between any pair of parties. In fact, recently, it is shown that one can achieve BA efficiently even without secure channels in a completely anonymous setting [26] hence removing the requirement for pairwise secure channels (but note that this latter work relies on proofs of work modeled in the RO model).

Why previously known techniques do not work. To motivate further our approach it is worth-while to emphasize in which way previous works fail to attain end-to-end verifiability in the standard model. Helios, culminates a long line of previous schemes that employ homomorphic type of voting $[17,22]$ and utilizes the Benaloh challenge [5] as the fundamental mechanism to attain verifiability. Helios by design requires the voter to utilize a voter supporting device to prepare a ciphertext and after an indeterminate number of trials, the voter will cast the produced ciphertext. Such ciphertexts are to be homomorphically tallied and thus they should be accompanied by a proof of proper computation. While such proofs are easy to construct based on e.g., [20], they can only be argued interactively (which is insufficient in our setting since a corrupt EA together with a corrupt voter may cook up a malformed proof that is indistinguishable from a proper one) or using a NIZK [10]. This latter approach is taken in Helios where a RO-based NIZK is utilized. In case the RO is dropped in favor of a standard model NIZK, security would be impossible in our model as NIZK's require a common reference string (CRS) and this is unavailable in the standard model; if the CRS is setup by the EA then in case it is malicious it will know and exploit the trapdoor; on the other hand, the voters are not interacting with each other and hence cannot setup the CRS by employing an MPC protocol. It follows that obtaining E2E verifiability in the standard model is impossible to overcome for Helios or any other similar scheme. On the other hand, in the case of Remotegrity/Scantegrity $n$ coins need to be obtained from the randomness beacon in order to prove the result correct. It is easy to verify that the system is insecure in terms of end-to-end verifiability in case the randomness beacon is biased. As before, the only parties active are the EA and the voters who cannot implement a randomness beacon that is required in the construction. In light of the above our construction offers a new paradigm in e-voting design: the randomness for the verification of the election can be collected distributively from the voters. Given that such randomness is by nature very weak (humans are very bad "randomness generators" and even worse malicious voters may collaborate with the election authority to cancel the honest voters' random bits) we show how suitable cryptographic techniques that deal with imperfect randomness can be employed to prove security.

Distributing the Election Authority. In our security model, we consider the EA as a single entity that is malicious in the verifiability game and honest in the privacy game. In practice one may want to distribute the EA to a number of "trustees" that collectively implement the EA functionality to improve the resiliency of the privacy property. While this is not a prime focus of our work (which centers on verifiability), it is feasible to design an efficient threshold 
protocol for implementing the EA. Note that our notion of voter privacy and receipt freeness can be easily extended to allow corrupted sub-authorities.

Other required properties of election systems. Our work by no means solves the complete set of desired requirements that are needed in an election system. Our voter-privacy definition implies receipt-freeness, i.e., provided that the voter receives the voter secret-key over an untappable channel ${ }^{2}$, the voter cannot convince any third party about the way she voted. Nevertheless, this does not imply coercion resistance as the voter may still be forced to divulge the voter secret prior to her ballot-casting (this does not violate voter privacy - it just prevents the voter from actually using the system and enables the adversary to vote on the voter's behalf). There are techniques that can be used to increase coercion resistance for internet-voting (e.g., those of $[29,16]$ and others) and they are compatible with our construction. We leave the integration of these techniques with information theoretic E2E verifiability for future work. Similarly, usability aspects are not within our current scope; nevertheless, we stress that we have implemented our scheme for 1-out-of- $m$ elections and we have used it in real-world experiments ${ }^{3}$.

Related work. In [11], Chaum suggested for the first time that anonymous communication can lead to voting systems with individual verifiability, i.e., the voters can verify that their votes were counted correctly. In [41], Sako and Killian introduce explicitly the notion of universal verifiability, that is, the ability for anyone to verify that the election result derives from the cast votes. Universal verifiability is also defined by Juels, Catalano and Jakobsson in [29] in the computational model assuming a trusted setup. Kremer, Ryan and Smyth [31] introduced symbolic definitions for individual and universal verifiability in the context of applied pi calculus. A formal definition of universal verifiability is also provided by Chevallier-Mames et al. in [15].

End-to-end verifiability in the sense of cast-as-intended, recorded-as-cast, tallied-as-recorded was an outcome of the works of Chaum [13] and Neff [38]. The novelty was the generation of receipts that could be used for simple voter verification while achieving privacy. The term of E2E verifiability (more precisely, E2E integrity) also appeared in [19]. Marneffe, Pereira and Quisquater presented an ideal-world definition for election systems in [23] without explicitly considering verifiability as a property of the ideal world. In [39], Popoveniuc et $a l$. proposed a definition of E2E verifiability via a list of properties.

Küsters, Truderung and Vogt [32] introduced symbolic and computational definitions of verifiability parameterized by a goal and an adversarial environment. In [34], the same authors showed that individual verifiability and universal verifiability are not sufficient to guarantee the "global" verifiability of an e-voting system. A number of other e-voting systems in the cryptographic setting that do not explicitly deal with E2E verifiability include $[18,7,21,22]$.

\footnotetext{
${ }^{2}$ An untappable channel enables the voter to deny the information that was transmitted in it. Physically distributing voter's secrets or using non-committing encryption [4] achieves untappability.

${ }^{3}$ For more information check our web-site http://www.demos-voting.org
} 
Benaloh and Fischer [18] provided a computational definition of privacy as the property that any coalition of malicious voters cannot distinguish between any two vote assignments coming from a subset of honest voters that have the same partial tally. Receipt-freeness has been first studied by Benaloh and Tuinstra [6] and described as the property of an e-voting system to generate fake voter transcripts that are indistinguishable from genuine transcripts. Following this logic, in our voter privacy/receipt-freenes definition, we require simulationbased indistiguishability of the views of the voters when they engage in the ballot-casting stage. Chevallier-Mames et al. [15] introduced definitions for unconditional of privacy and receipt-freeness and showed incompatibility results of universal verifiability with each of these two properties.

Formal definitions for privacy and receipt-freeness have been proposed in the context of applied pi calculus [24] and the universal composability model $[27,37]$. Küsters, Truderung and Vogt [33], mention that simulation-based definitions are often too strong to show security for reasonable e-voting systems, due to their "yes or no" nature (the real and ideal setting are either indistinguishable or not). In [34], they measure the level of privacy of an e-voting system w.r.t. to the observation power the adversary has in a protocol run, via a definition which is close to the Dolev-Yao model.

In [8], Bernhard et al. proposed a game-based notion of ballot privacy and study the privacy of Helios. In their model, an adversary that chooses a fixed vote $E$, cannot distinguish a bulletin board that contains ballots for real votes from a bulletin board that contains ballots for $E$. Their definition was extended by Bernhard, Pereira and Warinschi [9] by allowing the adversary to statically corrupt election authorities. Both these definitions, although they imply a strong inditinguishability property, do not consider receipt-freeness. We note that our game-based definition captures both privacy and receipt-freeness while restricted to a single EA (and it can easily be extended by including a set of trustees that the adversary may corrupt).

As we have mentioned previously, modelling coercion resistance is out of the scope of this work. We refer the reader to $[29,24,43,33]$ for formal definitions of coercion resistance in the cryptographic, symbolic and universal composability model.

Organization. In Section 2, we introduce the syntax and define the correctness, E2E verifibiality and voter privacy/receipt freeness of an e-voting system. In Section 3, we present at length the construction of our e-voting system, including a detailed description of all tools that are applied. In Section 4, we prove the $\mathrm{E} 2 \mathrm{E}$ verifibiality and voter privacy/receipt freeness of our e-voting system in the security framework of Section 2.

\section{E-voting Systems}

\subsection{Preliminaries}

We use $\lambda$ as the security parameter. Associated with an e-voting system, we also consider two other parameters, the number of voters $n$ and number of candidates 
$m$ which are both thought as polynomial functions of $\lambda$. Let $\Pi$ be an e-voting system, where $\mathcal{P}=\left\{P_{1}, \ldots, P_{m}\right\}$ is the set of candidates and $\mathcal{V}=\left\{V_{1}, \ldots, V_{n}\right\}$ is the set of voters. We denote by $\mathcal{U} \subseteq 2^{\mathcal{P}}$ the collection of subsets of candidates that the voters are allowed to choose to vote for (which may include a "blank" option too). The candidate selection $\mathcal{U}_{\ell}$ of voter $V_{\ell}$ is an element in $\mathcal{U}$.

Let $\mathcal{P}^{*}$ be the set of vectors of candidate selections of arbitrary length. Let $f$ be the election evaluation function from $\mathcal{P}^{*}$ to the set $\mathbb{Z}_{+}^{m}$ so that $f\left(\mathcal{U}_{1}, \ldots, \mathcal{U}_{n}\right)$ is equal to an $m$-vector whose $i$-th location is equal to the number of times $P_{i}$ was chosen in the candidate selections $\mathcal{U}_{1}, \ldots, \mathcal{U}_{n}$.

The entities involved in an e-voting system $\Pi$, are the voters $V_{1}, \ldots, V_{\ell}$, the election authority (EA) and the Bulletin Board (BB).

\subsection{Syntax and Correctness}

An e-voting system $\Pi$ is a quintuple of algorithms and protocols $\langle$ Setup, Cast, Tally, Result, Verify $\rangle$ specified as follows:

- The algorithm $\operatorname{Setup}\left(1^{\lambda}, \mathcal{P}, \mathcal{V}, \mathcal{U}\right)$ is executed by the EA and generates a master secret key $m s k, \Pi$ 's public parameters Pub (which include $\mathcal{P}, \mathcal{V}, \mathcal{U}$ ) and the voters' secrets $s_{1}, \ldots, s_{n}$. EA has a state, st which is initialized as $m s k$. In addition, it posts an initial public transcript $\tau=$ Pub on the BB.

- The interactive protocol Cast is between three parties, the voter $V_{\ell}$, the $\mathrm{BB}$ and the EA. $V_{\ell}$ has input $\left(\mathrm{Pub}, s_{\ell}, \mathcal{U}_{\ell}\right)$, EA has input $m s k$ and $\mathrm{BB}$ has input $\tau$. EA updates its state st and BB updates the public transcript $\tau$. Upon successful termination, the voter $V_{\ell}$ receives a receipt $\alpha_{\ell}$. We denote by view $w_{\ell}$ the view of the voter $V_{\ell}$ in the protocol Cast.

- The interactive protocol Tally with common input Pub is executed by the $\mathrm{EA}$ and the $\mathrm{BB}$ on inputs $m s k, \tau$ respectively. Upon successful termination, the BB updates the public transcript $\tau$.

- The algorithm $\operatorname{Result}(\tau)$ outputs the result $R_{\tau}$ for the election or returns $\perp$ in case such result is undefined.

- The algorithm $\operatorname{Verify}(\tau, \alpha)$ outputs a value in $\{0,1\}$, where $\alpha$ is a voter receipt (that corresponds to the voter's output from the Cast protocol).

Remark. In many election systems, the EA is implemented by more than a single authority. This means that Setup might be a protocol executed by those parties (as opposed to a standalone algorithm). However, from the point of view of E2E verifiability (where the system is considered malicious as a whole) this is completely immaterial. Hence, for simplicity in the syntax above we consider EA a single entity. In our construction the EA may also be distributed. We defer the details for how this may be done to the full version of the paper.

Definition 1 (Correctness). The e-voting system $\Pi$ has (perfect) correctness, if for any honest execution of $\Pi$ that results in a public transcript $\tau$ where the voters $V_{1}, \ldots, V_{n}$ cast votes for options $\mathcal{U}_{1}, \ldots, \mathcal{U}_{n}$ and received receipts $\alpha_{1}, \ldots, \alpha_{n}$, it holds that

$$
\operatorname{Result}(\tau)=f\left(\mathcal{U}_{1}, \ldots, \mathcal{U}_{n}\right) \text { and } \wedge_{\ell=1}^{n}\left(\operatorname{Verify}\left(\tau, \alpha_{\ell}\right)=1\right)
$$




\subsection{E2E Verifiability}

In order to define E2E verifiability formally, we introduce a suitable notation; given that candidate selections are elements of a set of $m$ choices, we may encode them as $m$-bit strings, where the bit in the $i$-th position is 1 if and only if candidate $P_{i}$ is selected. Further, we may aggregate the election results as the list with the number of votes each candidate has received, thus the output of the Result algorithm is a vector in $\mathbb{Z}_{+}^{m}$. In this case, a result is feasible if and only if the sum of all its coordinates is no greater than the number of voters.

In our formalization of the E2E verifiability, we postulate the existence of a vote extractor algorithm $\mathcal{E}$ (not necessarily running in polynomial-time) that explains the election transcript: namely, it receives input of the form $(\tau, A)$ where $\tau$ is an election transcript and $A=\left\{\alpha_{\ell}\right\}_{\ell \in \tilde{\mathcal{V}}}$ is a set of Cast protocol receipts. By $\tilde{\mathcal{V}}$, we denote the set of honest voters that voted successfully. Given such input, $\mathcal{E}$ will compute $n-|\tilde{\mathcal{V}}|$ vectors $\left\langle\mathcal{U}_{\ell}\right\rangle_{V_{\ell} \in \mathcal{V} \backslash \tilde{\mathcal{V}}}$ in $\{0,1\}^{m}$ (which correspond to the choices of all the voters outside of $\tilde{\mathcal{V}}$ ) that can be either a candidate selection if the voter has voted adversarially or a zero vector if the voter has not voted successfully; $\mathcal{E}$ returns the symbol $\perp$ in case such values cannot be defined. In the special case where all voters are honest and have voted successfully (i.e., $\tilde{\mathcal{V}}=\mathcal{V}$ ), $\mathcal{E}$ returns no value (outputs the empty set). The purpose of the $\mathcal{E}$ algorithm will be to capture the setting when the election transcript $\tau$ contains (in potentially encoded form) a set of well-formed actual votes.

Using the above notion, we will be capable to express the actual result encoded in an election transcript. Next, we want to formally express a measure of deviation from the actual election result (as such deviation is the objective of the adversary in an E2E verifiability attack). Some preliminary notions will be needed. In order to express formally the deviation the adversary aims at, it is natural to equip the space of results with a metric. We use the metric derived by the 1-norm, $\|\cdot\|_{1}$ scaled to half, i.e., $\mathrm{d}_{1}: \mathbb{Z}_{+}^{m} \times \mathbb{Z}_{+}^{m} \longrightarrow \mathbb{R}$ with $\mathrm{d}_{1}\left(w, w^{\prime}\right)=\frac{1}{2} \cdot\left\|w-w^{\prime}\right\|_{1}=\frac{1}{2} \cdot \sum_{i=1}^{n}\left|w_{i}-w_{i}^{\prime}\right|$ where $w_{i}, w_{i}^{\prime}$ is the $i$-th coordinate of $w, w^{\prime}$ respectively.

Consider $R \in \mathbb{Z}_{+}^{m}$ be the election results that correspond to the true voter intent of $n$ voters, and $R^{\prime} \in \mathbb{Z}_{+}^{m}$ be the published election results. Denote by $\max (\mathcal{U})$, the maximum cardinality of an element in $\mathcal{U}$. Two encodings of candidate selections are within $\max (\mathcal{U})$ distance, so intuitively, if the adversary wants to present $R^{\prime}$ as the result of the election, it may do that by manipulating the votes of at least $\mathrm{d}_{1}\left(R, R^{\prime}\right) / \max (\mathcal{U})$ voters.

We define next the E2E Verifiability game, $G_{\mathrm{E} 2 \mathrm{E}-\text { Int }}^{\mathcal{A}, \mathcal{E}, d, \theta}$, between the adversary $\mathcal{A}$ and a challenger $\mathcal{C}$ using a voter extractor $\mathcal{E}$, that takes as input the security parameter, $\lambda$, the number of candidates, $m$ and the number of voters, $n$.

Overview of the game $G_{\mathrm{E} 2 \mathrm{E}-\mathrm{Ver}}^{\mathcal{A}, \mathcal{E}, d, \theta}\left(1^{\lambda}, m, n\right)$. The attack game is parameterized by $d$, which is the deviation amount (according to the metric $\mathrm{d}_{1}(\cdot, \cdot)$ ) that the adversary wants to achieve and $\theta$, the minimum number of voters that $\mathcal{A}$ must allow to vote honestly and terminate successfully. The adversary starts by selecting the voter and candidate identities for given parameters $n, m$. It also determines the allowed ways to vote as described by the set $\mathcal{U}$. The adversary 
fully controls the EA. The adversary manages the Cast protocol executions where it assumes the role of the EA. For each voter, the adversary may choose to corrupt it or to allow the challenger to play on its behalf. In the second case, the adversary provides the candidate selection that the honest voter will use in the Cast protocol. The adversary completes the execution of EA which results to the complete election transcript published in the BB. The adversary will win the game provided that all $\theta$ honest voters that completed the Cast protocol successfully will also audit the result successfully but the deviation of the tally is at least $d$; the adversary will also win in case the extractor fails to produce the candidate selection of the dishonest voters (but $\theta$ honest voters still verify correctly). The attack game is specified in detail in Figure 1.

E2E Verifiability Game $G_{\mathrm{E} 2 \mathrm{E}-\mathrm{Ver}}^{\mathcal{A}, \mathcal{E}, \boldsymbol{\theta}}\left(1^{\lambda}, m, n\right)$

1. $\mathcal{A}$ chooses a list of candidates $\mathcal{P}=\left\{P_{1}, \ldots, P_{m}\right\}$, a set of voters $\mathcal{V}=\left\{V_{1}, \ldots, V_{n}\right\}$ and the set of allowed candidate selections $\mathcal{U}$. It provides $\mathcal{C}$ with the sets $\mathcal{P}, \mathcal{V}, \mathcal{U}$ along with information Pub and voter credentials $\left\{s_{\ell}\right\}_{\ell \in[n]}$. Throughout the game, $\mathcal{C}$ plays the role of the $\mathrm{BB}$.

2. The adversary $\mathcal{A}$ and the challenger $\mathcal{C}$ engages in an interaction where $\mathcal{A}$ schedules the Cast protocols of all voters. For each voter $V_{\ell}, \mathcal{A}$ can either completely control the voter or allow $\mathcal{C}$ to operate on their behalf, in which case $\mathcal{A}$ provides a candidate selection $\mathcal{U}_{\ell}$ to $\mathcal{C}$. Then, $\mathcal{C}$ engages with the adversary $\mathcal{A}$ in the Cast protocol so that $\mathcal{A}$ plays the role of EA. Provided the protocol terminates successfully, $\mathcal{C}$ obtains the receipt $\alpha_{\ell}$ on behalf of $V_{\ell}$.

Let $\tilde{\mathcal{V}}$ be the set of honest voters (i.e., those controlled by $\mathcal{C}$ ) that terminated successfully.

3. Finally, $\mathcal{A}$ posts the election transcript $\tau$ to the BB.

The game returns a bit which is 1 if and only if the following conditions hold true:

(i). $|\tilde{\mathcal{V}}| \geq \theta$, (i.e., at least $\theta$ honest voters terminated).

(ii). $\forall \ell \in[n]:$ if $V_{\ell} \in \tilde{\mathcal{V}}$, then $\operatorname{Verify}\left(\tau, \alpha_{\ell}\right)=1$ (i.e., the voters in $\tilde{\mathcal{V}}$ verify their ballot successfully).

and either one of the following two conditions:

(iii-a). If $\perp \neq\langle\mathcal{U}\rangle_{V_{\ell} \in \mathcal{V} \backslash \tilde{\mathcal{V}}} \leftarrow \mathcal{E}\left(\tau,\{\alpha\}_{V_{\ell} \in \tilde{V}}\right)$,

then

$$
\mathrm{d}_{1}\left(\boldsymbol{\operatorname { R e s u l t }}(\tau), f\left(\left\langle\mathcal{U}_{1}, \ldots, \mathcal{U}_{n}\right\rangle\right)\right) \geq d .
$$

(iii-b). $\perp \leftarrow \mathcal{E}\left(\tau,\left\{\alpha_{\ell}\right\}_{V_{\ell} \in \tilde{V}}\right)$.

Fig. 1: The E2E Verifiability Game between the challenger $\mathcal{C}$ and the adversary $\mathcal{A}$ using the vote extractor $\mathcal{E}$. 
Definition 2 (E2E-Verifiability). Let $0<\epsilon<1$ and $n, m, d, \theta \in \mathbb{N}$ with $d>0$ and $0<\theta \leq n$. The election protocol $\Pi$ w.r.t. the election function $f$ achieves $\mathrm{E} 2 \mathrm{E}$ verifiability with error $\epsilon$, for a number of at least $\theta$ honest successful voters and tally deviation $d$ if there exists a (not necessarily polynomial-time) voteextractor $\mathcal{E}$ such that for any adversary $\mathcal{A}$ :

$$
\operatorname{Pr}\left[G_{\mathrm{E} 2 \mathrm{E}-\operatorname{Ver}}^{\mathcal{A}, \mathcal{V}, \theta}\left(1^{\lambda}, m, n\right)=1\right] \leq \epsilon .
$$

In plain words, Definition 2 suggests that an E2E verifiable e-voting system, provides an "official explanation" of adversarial votes via the vote extractor $\mathcal{E}$, such that if at least $\theta$ voters verify the result, then any adversary that attempts to manipulate the election tally (that includes the honest votes and the official explanation of the adversarial votes) by a shift of $d$ votes will get caught except from some (supposedly small) probability $\epsilon$.

Remark. In the only previous works [34,32] where end-to-end verifiability was considered at a "global level" as we do here, it was expressed with respect to a set of "good" runs $\gamma$ of the e-voting protocol in the sense that a judge could test whether the protocol operated within the set $\gamma$. Even though sufficiently expressive, this formulation has the disadvantage that the set $\gamma$ remains undetermined and thus the level of verifiability that is offered by the definition hinges on the proper definition of $\gamma$ which may not be simple. Using our language the notion of a good run becomes explicit: a run of the e-voting protocol is good provided that the extractor $\mathcal{E}$ produces votes for the malicious voters which if they are added to the votes of the honest voters they produce a result that does not deviate from the published result according to the $d_{1}(\cdot, \cdot)$ metric. Note that our vote extractor may require super-polynomial time (in the same way that the set of good runs $\gamma$ may have a membership test of super-polynomial complexity). We remark that the use of a super-polynomial extractor to define properly the inputs of the malicious participants and hence the soundness of a multiparty protocol is not novel to our work. For example see, Micali, Pass and Rosen [36] where they used a similar construct to prove security of their general multiparty computation protocol.

\subsection{Voter Privacy (including Receipt-Freeness)}

The definition of voter privacy concerns the actions that may be taken by the adversary to break the privacy and learn some information about the candidate selections of the honest voters. We specify the goal of the adversary in a very general way. In particular, for an attack against voter privacy to succeed, we ask that there is an election result, for which the adversary is capable of distinguishing how the honest voters voted while it has access to (i) the actual receipts that the voters obtained after ballot-casting as well as (ii) a set of protocol views that are consistent with all the honest voters' views in the Cast protocol instances they participated (and the adversary has observed).

Observe that any system that is secure against such an attack scenario would possess also "receipt-freeness", i.e., voters cannot prove how they voted by showing the receipt they obtain from the Cast protocol or even presenting their view 
in the Cast protocol. Given that in the privacy definition we allow the adversary to observe the view of the voter in the Cast protocol, we need to allow the voter to be able to "lie" about her view in this protocol (otherwise an attack could be trivially mounted). Note that this would require the voter input to the Cast protocol to be delivered via an untappable channel; in particular, the adversary should not have any side-channel information about the voter's secrets $s_{1}, \ldots, s_{n}$.

We formally define the voter privacy of an election via a Voter Privacy/Receiptfreeness game, denoted by $G_{t \text {-priv }}^{\mathcal{A}, \mathcal{S}}$, that is played between an adversary $\mathcal{A}$ and a challenger $\mathcal{C}$, that takes as input the security parameter, $\lambda$, the number of voters, $n$, and the number of candidates, $m$, as described in Figure 2 and returns 1 or 0 depending on whether the adversary wins. An important feature of the game is the existence of an efficient "voter simulator" $\mathcal{S}$ that provides a simulated view of the voter in the Cast protocol. Intuitively, this simulator captures the way the voter can lie about her candidate selection in the Cast protocol in case she is coerced to present her view after she completes the ballot-casting procedure.

Overview of the game $G_{t \text {-priv }}^{\mathcal{A}, \mathcal{S}}\left(1^{\lambda}, n, m\right)$. The adversary starts by selecting the voters and candidates for given parameters $n, m$. It also determines the allowed ways to vote. The challenger flips a coin $b$ (that will change its behavior during the course of the game) and will perform the Setup protocol. Subsequently, the adversary will schedule all Cast protocols selecting which voters it prefers to corrupt and which ones it prefers to allow to vote honestly. The adversary is allowed to corrupt at most $t$ voters. The voters that remain uncorrupted are operated by the challenger and they are given two candidate selections to choose. The challenger will select which of the two candidate selections the voter will use in the Cast protocol according to the bit $b$. The adversary will also receive the receipt that is obtained by each voter as well as either the actual view of each voter during the Cast protocol, if $b=0$, or a simulated view, if $b=1$ (this addresses the receipt-freeness aspect). Upon completion of ballotcasting, the challenger executes the Tally protocol and posts the election result. Subsequently the adversary will attempt to guess $b$. The attack is successful provided that the adversary has corrupted up to $t$ voters, the election tally is the same with respect to the two alternatives provided for each honest voter by the adversary and the adversary manages to guess the challenger's bit $b$. The game is presented in more detail in figure 2.

Definition 3 (Voter Privacy/Receipt-Freeness). Let $n, m \in \mathbb{N}$. The $e$ voting system $\Pi$ w.r.t. the election function $f$ achieves voter privacy/receiptfreeness for at most $t$ corrupted voters, if there is a PPT voter simulator $\mathcal{S}$ such that for any PPT adversary $\mathcal{A}$ :

$$
\left|\operatorname{Pr}\left[G_{t-\operatorname{Priv}}^{\mathcal{A}, \mathcal{S}}\left(1^{\lambda}, n, m\right)=1\right]-1 / 2\right|=\operatorname{negl}(\lambda)
$$


Voter Privacy/Receipt-freeness Game $G_{t \text {-priv }}^{\mathcal{A}, \mathcal{S}}\left(1^{\lambda}, n, m\right)$

1. $\mathcal{A}$ on input $1^{\lambda}, n, m$, chooses a list of candidates $\mathcal{P}=\left\{P_{1}, \ldots, P_{m}\right\}$, a set of voters $\mathcal{V}=\left\{V_{1}, \ldots, V_{n}\right\}$, and the set of allowed candidate selections $\mathcal{U}$. It provides $\mathcal{C}$ the sets $\mathcal{P}, \mathcal{V}$, and $\mathcal{U}$.

2. $\mathcal{C}$ flips a coin $b \in\{0,1\}$ and performs the Setup protocol on input $\left(1^{\lambda}, \mathcal{P}, \mathcal{V}, \mathcal{U}\right)$ to obtain $m s k, s_{1}, \ldots, s_{n}, \mathrm{Pub}$; it provides $\mathcal{A}$ with Pub.

3. The adversary $\mathcal{A}$ and the challenger $\mathcal{C}$ engage in an interaction where $\mathcal{A}$ schedules the Cast protocols of all voters which may run concurrently. For each voter $V_{\ell} \in \mathcal{V}$, the adversary chooses whether $V_{\ell}$ is corrupted:

- If $V_{\ell}$ is corrupted, then $\mathcal{C}$ provides $s_{\ell}$ to $\mathcal{A}$, and then they engage in a Cast protocol where $\mathcal{A}$ plays the role of $V_{\ell}$ and $\mathcal{C}$ plays the role of EA and BB.

- If $V_{\ell}$ is not corrupted, $\mathcal{A}$ provides two candidate selections $\left\langle\mathcal{U}_{\ell}^{0}, \mathcal{U}_{\ell}^{1}\right\rangle$ to the challenger $\mathcal{C}$. $\mathcal{C}$ operates on $V_{\ell}$ 's behalf, using $\mathcal{U}_{\ell}^{b}$ as the $V_{\ell}$ 's input. The adversary $\mathcal{A}$ is allowed to observe the network trace of the Cast protocol where $\mathcal{C}$ plays the roles of $V_{\ell}, \mathrm{EA}$, and BB. When the Cast protocol terminates, the challenger $\mathcal{C}$ provides to $\mathcal{A}$ : (i) the receipt $\alpha_{\ell}$ that $V_{\ell}$ obtains from the protocol, and (ii) if $b=0$, the current view of the internal state of the voter $V_{\ell}$, view , that the challenger obtains from the Cast execution, or if $b=1$, a simulated view of the internal state of $V_{\ell}$ produced by $\mathcal{S}\left(\right.$ view $\left._{\ell}\right)$.

4. $\mathcal{C}$ performs the Tally protocol playing the role of $\mathrm{EA}$ and $\mathrm{BB}$. $\mathcal{A}$ is allowed to observe the network trace of that protocol.

5. Finally, $\mathcal{A}$ using all information collected above (including the contents of the $\mathrm{BB})$ outputs a bit $b^{*}$.

Denote the set of corrupted voters as $\mathcal{V}_{\text {corr }}$ and the set of honest voters as $\tilde{\mathcal{V}}=$ $\mathcal{V} \backslash \mathcal{V}_{\text {corr }}$. The game returns a bit which is 1 if and only if the following hold true:

(i). $b=b^{*}$ (i.e., the adversary guesses $b$ correctly).

(ii). $\left|\mathcal{V}_{\text {corr }}\right| \leq t$ (i.e., the number of corrupted voters is bounded by $t$ ).

(iii). $f\left(\left\langle\mathcal{U}_{\ell}^{0}\right\rangle_{V_{\ell} \in \tilde{\mathcal{V}}}\right)=f\left(\left\langle\mathcal{U}_{\ell}^{1}\right\rangle_{V_{\ell} \in \tilde{\mathcal{V}}}\right)$ (i.e., the election result w.r.t. the set of voters $\tilde{\mathcal{V}}$ does not leak $b)$.

Fig. 2: The Voter-privacy/Receipt-freeness game

\section{Presentation of Our e-Voting System}

Our system has three stages, setup, ballot-casting and tallying, that parallel the operation of a $\Sigma$ protocol. During setup stage, the EA produces a series of commitments and pre-audit data that correspond to a first move of a $\Sigma$ protocol that will establish the validity of the commitments. During ballot-casting, voters engage with the EA in a protocol that will result in the recording of their vote, as well as in the submission of a random coin flip that will be used to produce the challenge for the $\Sigma$ protocol. Voters will receive a receipt as their local output from the ballot-casting protocol that can be used for auditing the election 
result. In the third and final stage, the EA produces the tally of the election and completes the $\Sigma$ protocol by publishing openings to commitments as well as other necessary information needed for verification. The verification step can take place at any time after the completion of the process using a collection of at least one receipt from the ballot-casting stage.

In our system, the voter implementation during the ballot-casting stage is expressed as a probabilistic transducer (see e.g., [28]) with a communication tape that has a number of states polynomial in the number of candidates $m$ (and independent to other parameters such as $n, \lambda$ ). Given that such a machine is severely limited in the computational sense, in order to achieve ballot casting we utilize a code-voting approach (cf. [12]): the EA corresponds vote-codes to commitments posted in the BB, and voters cast their vote by simply sending to the EA the vote-code that they prefer. The commitments have an additive homomorphic property, hence it is possible to tally the result by homomorphically processing them and opening the resulting "tally commitment." The proof that we use in order to ensure verifiability is a conjunction of a cut-and-choose proof with a $\Sigma$ proof that a committed value belongs to a set. The challenge needed for the $\Sigma$ proof will be extracted by applying a suitable extraction mechanism to the coin flips of the voter transducers that are collected by the EA.

In Sections 3.1, 3.2, 3.3 and 3.4, we provide a detailed description of the tools that we apply for the construction of our system, i.e., (i) the homomorphic commitment scheme, (ii) a generalization of the Schwartz-Zippel lemma for imperfect randomness, (iii) the $\Sigma$ protocol and (iv) the challenge extraction mechanism, respectively. We describe our e-voting system in Section 3.5 and prove its correctness in Section 3.6. For the better understanding of our system, we provide a toy example in Section 3.7.

\subsection{Perfectly Binding Commitment}

To achieve integrity against computationally unbounded adversaries, we have to use a perfectly binding commitment scheme. Moreover, our system requires such a commitment scheme to be additively homomorphic to facilitate the tally and audit process. In this work, we instantiate the commitment scheme with lifted ElGamal over elliptic curves. We use elliptic curve domain parameters Param $:=(p, a, b, g, q)$ generated by the curve generator $\mathcal{G}\left(1^{\lambda}\right)$, consisting of a prime $p$ that specify the finite field $\mathbb{F}_{p}$, two elements $a, b \in \mathbb{F}_{p}$ that specify an elliptic curve $E\left(\mathbb{F}_{p}\right)$ defined by the equation: $E: y^{2}=x^{3}+a x+b(\bmod p)$, a base point $g=\left(x_{g}, y_{g}\right)$ on $E\left(\mathbb{F}_{p}\right)$, and a prime $q$ which is the order of $g$. We denote the cyclic group generated by $g$ as $\mathbb{G}$, and it is assumed that the DDH assumption holds over $\mathbb{G}$. More specifically, our commitment scheme consists of the following algorithms:

- Gen(Param, $\left.1^{\lambda}\right)$ : picks $x \leftarrow \mathbb{Z}_{q}$, sets $h:=g^{x}$, and outputs ck $:=($ Param, $h)$.

- $\operatorname{Com}_{\mathrm{ck}}(m ; r)$ : outputs $c:=\left(g^{r}, g^{m} h^{r}\right)$.

- $\operatorname{Ver}_{\text {ck }}(c ; m ; r)$ : outputs accept if $c=\left(g^{r}, g^{m} h^{r}\right)$; otherwise, outputs reject. 
It is obvious that the above commitment scheme is perfectly binding and computationally hiding under the DDH assumption, i.e. for any PPT adversary $\mathcal{A}$, we have that the advantage

$$
\operatorname{Adv}_{\text {hide }}(\mathcal{A}):=\operatorname{Pr}\left[\begin{array}{l}
\text { Param } \leftarrow \mathcal{G}\left(1^{\lambda}\right) ; \mathrm{ck} \leftarrow \operatorname{Gen}\left(\operatorname{Param}, 1^{\lambda}\right) ; \\
\left(m_{0}, m_{1}\right) \leftarrow \mathcal{A}(\operatorname{Param}, \mathrm{ck}) ; b \leftarrow\{0,1\} ; \\
r \leftarrow \mathbb{Z}_{q}: \mathcal{A}\left(\operatorname{Com}_{\mathrm{ck}}\left(m_{b} ; r\right)\right)=b
\end{array}\right]-1 / 2 \mid
$$

is negligible in $\lambda$. The commitment scheme is additively homomorphic. Namely,

$$
\operatorname{Com}_{\mathrm{ck}}\left(m_{1} ; r_{1}\right) \cdot \operatorname{Com}_{\mathrm{ck}}\left(m_{2} ; r_{2}\right)=\operatorname{Com}_{\mathrm{ck}}\left(m_{1}+m_{2} ; r_{1}+r_{2}\right) .
$$

\subsection{Schwartz-Zippel (min-entropy variant)}

We need a min-entropy variant of the Schwartz-Zippel lemma, to check the equality of two univariate polynomials $f_{1}, f_{2}$, i.e. test $f_{1}(x)-f_{2}(x)=0$ for random $x \stackrel{D}{\leftarrow} \mathbb{Z}_{q}$. The probability that the test passes is at most $\frac{\max \left(d_{1}, d_{2}\right)}{2^{\kappa}}$ if $f_{1} \neq f_{2}$, where $d_{i}$ is the degree of $f_{i}$ for $i \in\{1,2\}$. We state the following lemma without proof (a proof will be provided in the full version).

Lemma 1 (min-entropy Schwartz-Zippel). Let $f(x)$ be a non-zero univariate polynomial of degree $d$ over $\mathbb{Z}_{q}$. Let $D$ be a probability distribution on $\mathbb{Z}_{q}$ such that $H_{\infty}(D) \geq \kappa$. The probability of $f(x)=0$ for a randomly chosen $x \stackrel{D}{\leftarrow} \mathbb{Z}_{q}$ is at most $\frac{d}{2^{\kappa}}$.

\subsection{A $\Sigma$ Protocol for Candidate Encoding Correctness}

In order to present the $\Sigma$ protocol with clarity, we outline some necessary excerpts of the description of our system that will be explained in detail in Section 3.5.

Let $N=n+1$, where $n$ is the number of voters. Each voter is given a ballot that consists of two equivalent parts that contain a list of $m$ vote-codes corresponding to the list candidates $\left\{P_{1}, \ldots, P_{m}\right\}$. The voter will flip a coin to choose the part she is going to use for voting. At the Setup phase, each ballot is posted to the BB in committed form. Namely, it consists of two sets of commitments $E_{\ell, j}^{(a)}$ for $a \in\{0,1\}, \ell=1, \ldots, n, j=1, \ldots, m$, and each set commits to a permutation of the encoded candidates, where candidate $P_{j}$ is encoded as $N^{j-1}$.

We emphasize that it is not necessary to prove that each set of the commitments commits to a permutation of the encoded candidates $\left\{N^{0}, \ldots, N^{m-1}\right\}$ in an 1-out-of- $m$ election. This is due to two facts: (i) EA will open one of the two sets of commitments according to the corresponding voter's coin $a_{\ell}$ (the set that corresponds to the unused ballot part); therefore, a malicious EA will be caught with probability $1 / 2$ by each honest voter if any of the committed sets is not a permutation of the encoded candidates or is an inconsistent permutation of the encoded candidates w.r.t. the one on the voter's ballot. (ii) Even if we ensure 
that the set of the commitments commits to a permutation of the encoded candidates, it does not imply that the permutation is consistent to the one on the voter's ballot. In an 1-out-of- $m$ election, only one of the commitments will be used for tally, and thus proving that the set of the commitments commits to an unknown permutation of the encoded candidates can only provide the guarantee that the tallied commitment commits to an encoded candidate. Note that this guarantee is important; otherwise, given that we perform homomorphic tallying, it may be feasible for a cheating EA to introduce a large deviation to the actual tally result via a single inconsistent ballot; for instance, EA may commit to $10000 \cdot N^{j-1}$ for some $j \in[m]$. Hence, we want the EA to show that each commitment commits to one of $N^{j-1}$ for $j \in[m]$. ${ }^{4}$ We can formalize the correctness of a single commitment problem as follows. Given commitments $E$, the prover wants to convince the verifier that he knows $r \in \mathbb{Z}_{q}$ such that $E=\operatorname{Com}_{\mathrm{ck}}\left(N^{i} ; r\right)$ and $i \in[0, m-1]$. Let $i, r$ be the prover's private input, and w.l.o.g. we assume $m$ is a perfect power of 2 . For general cases, say $2^{e-1} \leq m \leq 2^{e}$, we can show the conjunction $i \in\left[0,2^{e}\right] \wedge\left(i+2^{e}-m\right) \in\left[0,2^{e}\right]$. Our $\Sigma$ Protocol is described in Fig. 3.

Theorem 1. Let $N>0$ be a public integer. Given common input $E \in \mathbb{G} \times \mathbb{G}$, the protocol described in Fig. 3 is a $\Sigma$ protocol for knowledge of $i \in \mathbb{N}, r \in \mathbb{Z}_{q}$ such that $E=\operatorname{Com}_{\mathrm{ck}}\left(N^{i} ; r\right), i \in[0, m-1]$ that is perfectly complete, statistically sound with soundness error $2^{-\kappa+1+\log \log m}$ when the verifier's challenge has min-entropy $\kappa$ and computationally zero-knowledge with distinguishing advantage $\operatorname{Adv}_{\mathrm{zk}}(\mathcal{A}) \leq \log m \cdot \operatorname{Adv}_{\text {hide }}(\mathcal{A})$ for any PPT adversary $\mathcal{A}$.

Proof. It is straightforward to check that protocol in Fig. 3 achieves perfect completeness.

In terms of statistical soundness, the protocol verifies two facts. Namely, (i) $\left\{B_{j}\right\}_{j \in[0, \log m-1]}$ commits to either 0 or 1 , and (ii) $E$ commits to $N^{\sum_{j=0}^{\log m-1} b_{j} 2^{j}}=$ $N^{i}$, where $b_{j}$ is the opening of $B_{j}$. To check the first fact, for each committed $b_{j}$ the protocol builds the degree 1 polynomial

$$
g_{1}(X)=\left(1-b_{j}\right)\left(b_{j} X+t\right)+c_{0}=\left(1-b_{j}\right) b_{j} X+c_{0}^{\prime}
$$

for some $c_{0}$ and $c_{0}^{\prime}$. By min-entropy Schwartz-Zippel Lemma 1, if $H_{\infty}(\rho) \geq \kappa$ and $g_{1}(\rho)=0$, the probability $\operatorname{Pr}\left[\left(1-b_{j}\right) b_{j} \neq 0\right] \leq 2^{-\kappa}$. Hence, with at least $1-2^{-\kappa}$ probability $\left(1-b_{j}\right) b_{j}=0$, which implies $b_{j} \in\{0,1\}$. To check the second fact, the protocol first computes $A_{j}=B_{j}^{N^{2^{j}}-1} \cdot \operatorname{Com}_{\mathrm{ck}}(1 ; 0)$ homomorphically. Let $a_{j}$ be the opening of $A_{j}$. It is easy to see that $a_{j}=N^{2^{j}}$ if $b_{j}=1, a_{j}=1$

\footnotetext{
${ }^{4}$ For efficiency, EA is only required to show the commitments that are used for tally commit to valid encoded candidates. On the other hand, since EA cannot predicate which commitments are going to be used for tally before the election, she has to prepare all the $\Sigma$ protocols in the Setup phase; whereas she is only required to complete those $\Sigma$ protocols for the commitment that will be tallied in the Tally phase.
} 


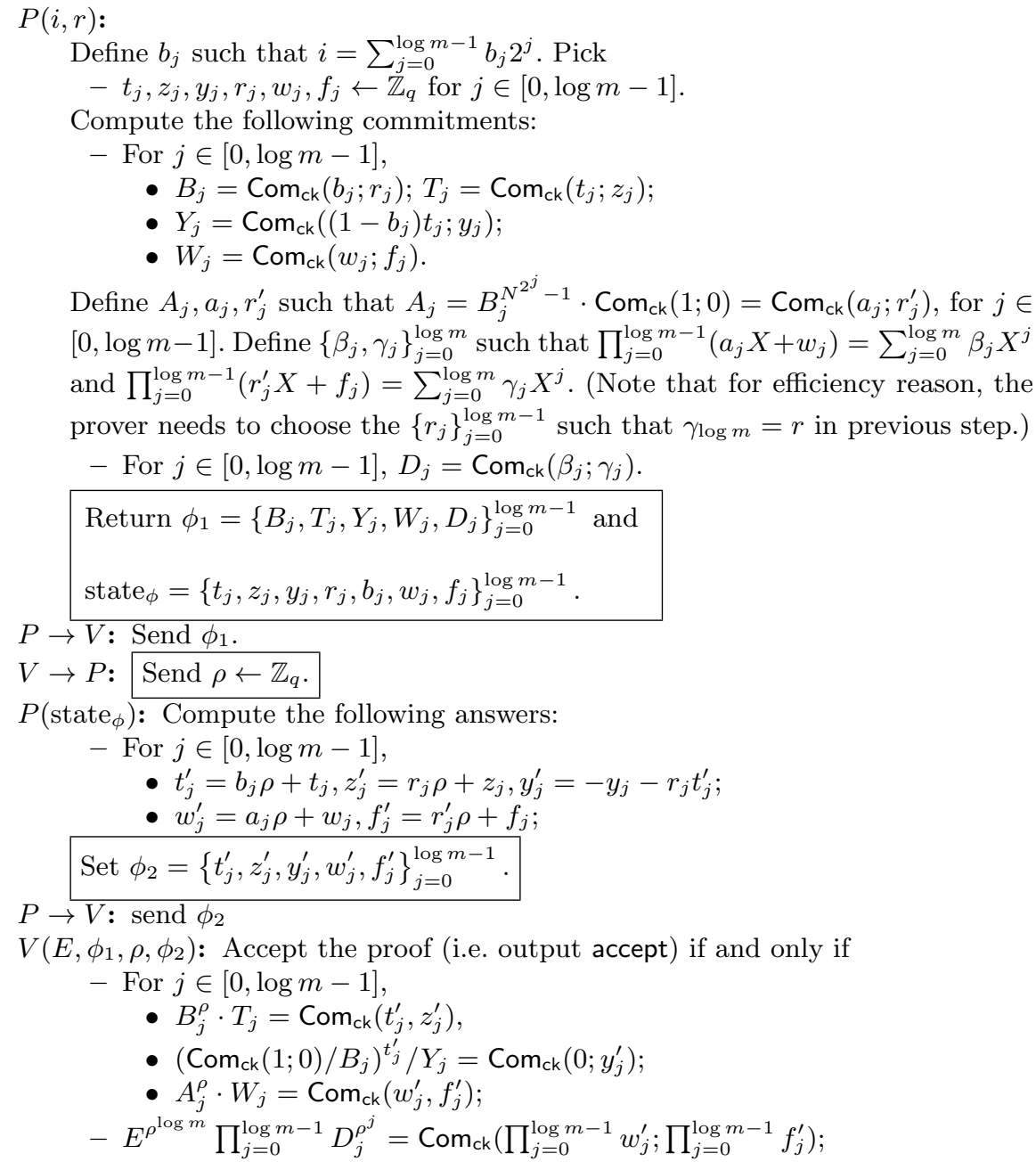

Define $A_{j}, a_{j}, r_{j}^{\prime}$ such that $A_{j}=B_{j}^{N^{2^{j}}-1} \cdot \operatorname{Com}_{\mathrm{ck}}(1 ; 0)=\operatorname{Com}_{\mathrm{ck}}\left(a_{j} ; r_{j}^{\prime}\right)$, for $j \in$ $[0, \log m-1]$. Define $\left\{\beta_{j}, \gamma_{j}\right\}_{j=0}^{\log m}$ such that $\prod_{j=0}^{\log m-1}\left(a_{j} X+w_{j}\right)=\sum_{j=0}^{\log m} \beta_{j} X^{j}$ and $\prod_{j=0}^{\log m-1}\left(r_{j}^{\prime} X+f_{j}\right)=\sum_{j=0}^{\log m} \gamma_{j} X^{j}$. (Note that for efficiency reason, the prover needs to choose the $\left\{r_{j}\right\}_{j=0}^{\log m-1}$ such that $\gamma_{\log m}=r$ in previous step.) - For $j \in[0, \log m-1], D_{j}=\operatorname{Com}_{\mathrm{ck}}\left(\beta_{j} ; \gamma_{j}\right)$.

Return $\phi_{1}=\left\{B_{j}, T_{j}, Y_{j}, W_{j}, D_{j}\right\}_{j=0}^{\log m-1}$ and state $_{\phi}=\left\{t_{j}, z_{j}, y_{j}, r_{j}, b_{j}, w_{j}, f_{j}\right\}_{j=0}^{\log m-1}$.

$P \rightarrow V:$ Send $\phi_{1}$.

$V \rightarrow P:$ Send $\rho \leftarrow \mathbb{Z}_{q}$.

$P\left(\right.$ state $\left._{\phi}\right)$ : Compute the following answers:

- For $j \in[0, \log m-1]$,

- $t_{j}^{\prime}=b_{j} \rho+t_{j}, z_{j}^{\prime}=r_{j} \rho+z_{j}, y_{j}^{\prime}=-y_{j}-r_{j} t_{j}^{\prime}$

- $w_{j}^{\prime}=a_{j} \rho+w_{j}, f_{j}^{\prime}=r_{j}^{\prime} \rho+f_{j}$;

Set $\phi_{2}=\left\{t_{j}^{\prime}, z_{j}^{\prime}, y_{j}^{\prime}, w_{j}^{\prime}, f_{j}^{\prime}\right\}_{j=0}^{\log m-1}$.

$P \rightarrow V:$ send $\phi_{2}$

$V\left(E, \phi_{1}, \rho, \phi_{2}\right)$ : Accept the proof (i.e. output accept) if and only if

- For $j \in[0, \log m-1]$,

- $B_{j}^{\rho} \cdot T_{j}=\operatorname{Com}_{\mathrm{ck}}\left(t_{j}^{\prime}, z_{j}^{\prime}\right)$,

- $\left(\operatorname{Com}_{\mathrm{ck}}(1 ; 0) / B_{j}\right)^{t_{j}^{\prime}} / Y_{j}=\operatorname{Com}_{\mathrm{ck}}\left(0 ; y_{j}^{\prime}\right)$

- $A_{j}^{\rho} \cdot W_{j}=\operatorname{Com}_{\mathrm{ck}}\left(w_{j}^{\prime}, f_{j}^{\prime}\right)$;

$-E^{\rho^{\log m}} \prod_{j=0}^{\log m-1} D_{j}^{\rho^{j}}=\operatorname{Com}_{\mathrm{ck}}\left(\prod_{j=0}^{\log m-1} w_{j}^{\prime} ; \prod_{j=0}^{\log m-1} f_{j}^{\prime}\right)$

Fig. 3: The $\Sigma$ Protocol for Ballot Correctness

if $b_{j}=0$, thus it holds that $a_{j}=b_{j} N^{2^{j}}+1-b_{j}=N^{b_{j} 2^{j}}$. So that the protocol just needs to verify that $E$ commits to the product of $a_{j}$ 's. The verifier checks equality between two degree $\log m$ polynomials

$$
g_{2}(X)=\prod_{j=0}^{\log m-1}\left(a_{j} X+w_{j}\right)=\sum_{j=0}^{\log m} \beta_{j} X^{j} \text { and } g_{2}^{\prime}(X)=u X^{\log m}+\sum_{j=0}^{\log m-1} \beta_{j}^{*} X^{j}
$$

where $u$ is the opening of $E$ and $\beta_{j}^{*}$ which is the opening of $D_{j}$ and are provided by the (potentially malicious) prover. By min-entropy Schwartz-Zippel lemma, if $H_{\infty}(\rho) \geq \kappa$ and $g_{2}(\rho)=g_{2}^{\prime}(\rho)$, the probability $\operatorname{Pr}\left[u=\beta_{\log m}\right] \geq 1-\frac{\log m}{2^{\kappa}}$. Hence, 
we have $u=N^{\sum_{j=0}^{\log m-1} b_{j} 2^{j}}$ with at least $1-\frac{\log m}{2^{\kappa}}$ probability conditioned on the fact (i). Given that all $b_{0}, \ldots, b_{\log m-1}$ need to be shown in $\{0,1\}$ the entire proof is statistically sound with probability $\left(1-2^{-\kappa}\right)^{\log m}\left(1-\frac{\log m}{2^{\kappa}}\right) \geq 1-\log m \cdot 2^{-\kappa+1}$.

Our protocol satisfies special soundness, i.e. there exists an extractor that can extract $i \in \mathbb{N}, r \in \mathbb{Z}_{q}$ if the prover is able to complete the protocol twice with the same $\phi_{1}$ but two distinct challenges (we omit the construction of the extractor).

To show special honest verifier zero-knowledge property, we now construct a simulator that on input $\hat{\rho} \in \mathbb{Z}_{q}$ can output a transcript that is indistinguishable from the real one. The simulator randomly picks $b_{0}, \ldots, b_{\log m-1} \leftarrow\{0,1\}$ and generates $\left\{t_{j}, z_{j}, y_{j}, r_{j}, B_{j}, T_{j}, Y_{j}, t_{j}^{\prime}, z_{j}^{\prime}, y_{j}^{\prime}, w_{j}, f_{j}, W_{j}, w_{j}^{\prime}, f_{j}^{\prime}\right\}_{j=0}^{\log m-1}$ according to the protocol description. It then generates $\left\{D_{j}\right\}_{j=1}^{\log m-1}$ according to the protocol and set

$$
D_{0}=\operatorname{Com}_{\mathrm{ck}}\left(\prod_{j=0}^{\log m-1} w_{j}^{\prime} ; \prod_{j=0}^{\log m-1} f_{j}^{\prime}\right) /\left(E^{\hat{\rho}^{\log m}} \prod_{j=1}^{\log m-1} D_{j}^{\hat{\rho}^{j}}\right)
$$

Subsequentely, the simulator sets $\hat{\phi}_{1}=\left\{B_{j}, T_{j}, Y_{j}, W_{j}, D_{j}\right\}_{j=0}^{\log m-1}$ and $\hat{\phi}_{2}=$ $\left\{t_{j}^{\prime}, z_{j}^{\prime}, y_{j}^{\prime}, w_{j}^{\prime}, f_{j}^{\prime}\right\}_{j=0}^{\log m-1}$, and it outputs $\left(\hat{\phi}_{1}, \hat{\rho}, \hat{\phi}_{2}\right)$. First of all, it is obvious that all the verification equations hold. Secondly, the distribution of all the variables in $\hat{\phi}_{2}$ are uniformly random, which is identical to that of a real transcript. Moreover, if the adversary can distinguish the simulated $\hat{\phi}_{1}$ from that of a real transcript, she must be able to distinguish at least one of the fake $\left\{B_{j}\right\}_{j=0}^{\log m-1}$. By hybrid argument, we have for any PPT adversary $\mathcal{A}$, the advantage to distinguish the simulated proof is $\operatorname{Adv}_{\mathrm{zk}}(\mathcal{A}) \leq \log m \cdot \operatorname{Adv}_{\text {hide }}(\mathcal{A})$.

\subsection{Producing the Verifier's Challenges}

The main difficulty in our setting is that we would like to extract the challenge of the $\Sigma$ protocol from the voters' coins $\boldsymbol{a}=\left\langle a_{1}, \ldots, a_{n}\right\rangle \in\{0,1\}^{n}$ using a deterministic algorithm. Recall that some of the voters might be malicious and colluding with the EA, so the entropy of the voters' coins is only contributed by the honest voters while the malicious voters' coins can depend on the honest ones. Note that the voters' coins should be ordered by their serial numbers, rather than their submission order. This is because in the latter case, the adversary can schedule the Cast protocols of all voters at will and as a result reduce the min-entropy of $\boldsymbol{a}$ to be at most $\log \theta$ where $\theta$ is the number of honest voters. Such level of entropy is insufficient to provide a sufficiently small verifiability error (that ideally drops exponentially with $\theta$ ). For all the uncast ballots, we set their corresponding coins to 0 by default; therefore, $\boldsymbol{a}$ is always an $n$-bit source, regardless of the number of voters that complete the Cast protocol. We observe that the voters' coins $\boldsymbol{a}$ is a weaker source compared to a non-oblivious bit-fixing source [30], as the adversary is able to choose which bit(s) to fix during the coin flipping (source generation) process. On the other hand, if we restrict 
the adversary $\mathcal{A}$ in our verifiability game from being capable of scheduling Cast protocols freely and all voters have to submit their votes sequentially according to a pre-determined order in the ballot casting stage, the source $\boldsymbol{a}$ can be viewed as an adaptive bit-fixing source [35]; in such case, we can employ the deterministic extractor construction framework from [30] which applies a deterministic low influence function on segments of the source. The majority function is proven to be an optimal low influence function thus in this way we obtain a deterministic extractor that generates the challenge. However, this adversarial setting is not realistic in practice as ballot casting might be scheduled adversarially. Nevertheless, we emphasise that even using a non-oblivious bit-fixing source, Kamp and Zuckerman showed that at most $n / \ell$ bits can be extracted when $\ell$ out of $n$ bits are fixed [30]. This result implies that if a deterministic extractor is used to generate $\Theta(\lambda)$ random bits, then this will restrict the percentage of corrupted voters to be below $\Theta\left(\frac{1}{\lambda}\right)$ which might also be not a realistic expectation in practice. An alternative approach may use a condenser as opposed to an extractor. Randomized condensers with a small/constant seed space have been put forth see e.g. [2,40]; using such a tool one may iterate over all possible seeds and thus be assured that one of the seeds will allow the condenser to produce a sufficiently random challenge. For instance, Barak et al. [2] proposed a basic 2-bit seed condenser con : $\{0,1\}^{n} \rightarrow\left(\{0,1\}^{n / 3}\right)^{4}$ such that for every $\delta$-source $X$ with $0<\delta<0.9$, at least one of the 4 output blocks of $\operatorname{con}(X)$ is a $\left(\delta+\Omega\left(\delta^{2}\right)\right)$-source. Based on the composing lemma (Lemma 5.5 [2]), we can iteratively apply the condenser to achieve any desired constant rate. Given a $c$ coin condenser Con : $\{0,1\}^{n} \mapsto\left(\{0,1\}^{\ell}\right)^{c}$, in order to produce a good challenge, by definition, it should hold that $c \cdot \ell>n$, which means that the condenser will produce $c$ blocks, one of which is guaranteed to be sufficiently random. However as we observe below, we can utilize ZK amplification to obtain essentially the same result as with a $c$-coin condenser while sacrificing very little entropy from the weak source. We explain our technique next.

Let $\{0,1\}^{\ell_{\Sigma}}$ be the challenge space, where $\ell_{\Sigma}=\lfloor\log q\rfloor$ and $q$ is the order of the underlying group used in the $\Sigma$ protocol. Assume $n / k \leq \ell_{\Sigma}$ for some $k \in \mathbb{Z}^{+}$. We evenly partition the voters' coins $\boldsymbol{a}$ into $k$ blocks, denoted by $\boldsymbol{a}_{1}, \ldots, \boldsymbol{a}_{k}$. For each block $\boldsymbol{a}_{i}$, the EA should prove the correctness of the ballots using a separate $\Sigma$ protocol with $\boldsymbol{a}_{i}$ as its challenge. The verifier only accepts the EA's proof if all the $\Sigma$ protocols are valid. The theorem below shows that the soundness error of this $k$-times repeated $\Sigma$ protocol drops exponentially with $\theta-k(\log \log m+1)$.

Theorem 2. Denote $\boldsymbol{a}=\left(\boldsymbol{a}_{1}, \ldots, \boldsymbol{a}_{k}\right)$, and suppose $H_{\infty}(\boldsymbol{a})=\theta$. For all adversarial prover $\mathcal{A}$, we have that

$$
\begin{aligned}
& \epsilon(m, n, k, \theta)=\operatorname{Pr}\left[\begin{array}{l}
\mathrm{ck} \leftarrow \operatorname{Gen}\left(\operatorname{Param}, 1^{\lambda}\right) ;\left(E, x, r,\left\{\phi_{1, i}\right\}_{i=1}^{k}\right) \leftarrow \mathcal{A}(\text { Param }, \mathrm{ck}) ; \\
\left\{\phi_{2, i}\right\}_{i=1}^{k} \leftarrow \mathcal{A}\left(\boldsymbol{a}_{1}, \ldots, \boldsymbol{a}_{k}\right): \operatorname{Ver}_{\mathrm{ck}}(E ; x ; r)=\text { accept } \wedge \\
x \notin\left\{N^{0}, \ldots, N^{m-1}\right\} \wedge \forall i \in[k], V\left(E, \phi_{1, i}, \boldsymbol{a}_{i}, \phi_{2, i}\right)=\text { accept }
\end{array}\right] \\
& \leq 2^{k \log \log m-\theta+k} .
\end{aligned}
$$

Proof. See full version of this paper. 


\subsection{Description of our e-voting system}

The description of our e-voting system follows the syntax in Section 2.2. For simplicity, we present our system for 1-out-of-m elections, i.e. $\mathcal{U}=\left\{\left\{P_{1}\right\}, \ldots,\left\{P_{m}\right\}\right\}$. The commitment scheme and the $\Sigma$-protocol that are applied in our system, are the ones presented at length in sections 3.1 and 3.3 respectively.

$\operatorname{Setup}\left(1^{\lambda}, \mathcal{P}=\left\{P_{1}, \ldots, P_{m}\right\}, \mathcal{V}=\left\{V_{1}, \ldots, V_{n}\right\}, \mathcal{U}=\left\{\left\{P_{1}\right\}, \ldots,\left\{P_{m}\right\}\right\}\right)$. Let (Gen, Com, Ver) be the PPT algorithms that constitute the perfectly binding, computationally hiding and additively homomorphic commitment scheme presented in Section 3.1. The EA runs Gen $\left(\operatorname{Param}, 1^{\lambda}\right)$ to generate the commitment key ck. Then, for $\ell \in[n]$, EA executes the following steps:

(i). It selects a unique label for the $\ell$-th double ballot denoted by $\operatorname{tag}_{\ell}$.

(ii). It selects random permutations $\pi_{\ell}^{(0)}, \pi_{\ell}^{(1)}$ over $[m]$. The use of $\pi_{\ell}^{(0)}$ (reps. $\left.\pi_{\ell}^{(1)}\right)$ is to shuffle the order that the (vote-code, candidate) pairs in the part $s_{\ell}^{(0)}$ (resp. $s_{\ell}^{(1)}$ ) of the double ballot $s_{\ell}$ will be posted on the BB (in committed form), in order to support privacy.

(iii). For $j \in[m]$, it selects unique vote-codes $C_{\ell, j}^{(0)}, C_{\ell, j}^{(1)} \leftarrow \mathbb{Z}_{q}$, where $q$ is the size of the group of the commitment scheme ${ }^{5}$. The vote-code $C_{\ell, j}^{(0)}$ (resp. $C_{\ell, j}^{(1)}$ ) is the one that will be associated with candidate $P_{j}$ in part $s_{\ell}^{(0)}$ (resp. $\left.s_{\ell}^{(1)}\right)$ of $s_{\ell}$.

(iv). For $a \in\{0,1\}$, it prepares the ballot part $s_{\ell}^{(a)}=\left\{\left(P_{j}, C_{\ell, j}^{(a)}\right)\right\}_{j \in[m]}$ and generates the ballot

$$
s_{\ell}=\left(\operatorname{tag}_{\ell}, s_{\ell}^{(0)}, s_{\ell}^{(1)}\right)
$$

(v). For $j \in[m]$, it computes $j^{\prime}=\pi_{\ell}^{(a)}(j)$ and

- For $a \in\{0,1\}$ (where $a$ indicates the part $s_{\ell}^{(a)}$ of $s_{\ell}$ ), it chooses randomness $t_{\ell, j^{\prime}}^{(a)} \leftarrow \mathbb{Z}_{q}$ and computes the vote-code commitment for $C_{\ell, j^{\prime}}^{(a)}$ :

$$
U_{\ell, j^{\prime}}^{(a)}=\operatorname{Com}_{\mathrm{ck}}\left(C_{\ell, j^{\prime}}^{(a)} ; t_{\ell, j^{\prime}}^{(a)}\right) \text {. }
$$

- For $a \in\{0,1\}$, it chooses randomness $r_{\ell, j^{\prime}}^{(a)} \leftarrow \mathbb{Z}_{q}$ and computes the encoded candidate commitment for $P_{j^{\prime}}$ :

$$
E_{\ell, j^{\prime}}^{(a)}=\operatorname{Com}_{\mathrm{ck}}\left((n+1)^{j^{\prime}-1} ; r_{\ell, j^{\prime}}^{(a)}\right),
$$

\footnotetext{
${ }^{5}$ For simplicity in presentation, we commit to the vote-codes using the homomorphic commitment scheme of Section 3.1. We stress that since no arithmetic operations are executed in the vote-code commitments, we could use more efficient commitment schemes and in this case vote-codes may be drawn from a domain that is smaller than $\mathbb{Z}_{q}$ resulting in a more "user-friendly" interface.
} 
where $(n+1)^{j^{\prime}-1}$ is the encoding of candidate $P_{j^{\prime}}$. This encoding is selected to ensure the correctness of our system, as we show in Theorem 3.

- For $a \in\{0,1\}$, EA prepares pre-audit data $\phi_{1, \ell, j^{\prime}}^{(a)}$ to be used for verifying that $E_{\ell, j^{\prime}}^{(a)}$ is a commitment to a valid encoding from the set $\left\{(n+1)^{0}, \ldots,(n+1)^{m-1}\right\}$ at the verification phase. In addition, it maintains prover state $\operatorname{state}_{\phi, \ell, j^{\prime}}^{(a)}$. Both $\phi_{1, \ell, j^{\prime}}^{(a)}$ and state $_{\phi, \ell, j^{\prime}}^{(a)}$ are described in the $\Sigma$-protocol shown in Figure 3 (first move) of Section 3.3.

(vi). $\mathrm{Pub}_{\ell}=\left(\operatorname{tag}_{\ell},\left\{\left(U_{\ell, j^{\prime}}^{(a)}, E_{\ell, j^{\prime}}^{(a)}, \phi_{1, \ell, j^{\prime}}^{(a)}\right)\right\}_{j \in[m]}^{a \in\{0,1\}}\right)$ is the public information w.r.t. $s_{\ell}$. It is indexed by $\operatorname{tag}_{\ell}$ and contains the ballot information for both parts in committed form, as well as the respective pre-audit data. The information that refers to the (vote-code, candidate) pair $\left(C_{\ell, j^{\prime}}^{(a)}, P_{j^{\prime}}\right)$ is tabulated in the $j$-th location of the part that is associated with $s_{\ell}^{(a)}$.

The public information that EA generates is

$$
\mathrm{Pub}=\left(\mathrm{ck}, \mathcal{P}, \mathcal{U},\left\{\mathrm{Pub}_{\ell}\right\}_{\ell \in[n]}\right) \text {. }
$$

The secret key of EA is

$$
m s k=\left\{\mathrm{Pub}_{\ell}, s_{\ell}, m s k_{\ell}, \operatorname{state}_{\phi, \ell}\right\}_{\ell \in[n]} \text {, }
$$

where we denote $m s k_{\ell}=\left\{\left(C_{\ell, j}^{(a)}, t_{\ell, j}^{(a)}, \pi_{\ell}^{(a)}(j)=j^{\prime}, r_{\ell, j}^{(a)}\right)\right\}_{j \in[m]}^{a \in\{0,1\}}$ and $\operatorname{state}_{\phi, \ell}=$ $\left\{\operatorname{state}_{\phi, \ell, j^{\prime}}^{(a)}\right\}_{j \in[m]}^{a \in\{0,1\}}$.

The Cast protocol. On input $\left(\mathrm{Pub}, s_{\ell}, \mathcal{U}_{\ell}\right)$, voter $V_{\ell}$ flips a coin $a_{\ell} \leftarrow\{0,1\}$ and picks part $s_{\ell}^{\left(a_{\ell}\right)}$ to vote and part $s_{\ell}^{\left(a_{\ell}\right)}$ for audit. Let $P_{j_{\ell}}$ be the candidate that $V_{\ell}$ is going to vote for, i.e., $\mathcal{U}_{\ell}=\left\{P_{j_{\ell}}\right\}$. Then, $V_{\ell}$ selects to submit $C_{\ell, j_{\ell}}^{\left(a_{\ell}\right)}$, which is the vote-code that corresponds to $P_{j_{\ell}}$ in part $s_{\ell}^{\left(a_{\ell}\right)}$. Next, $V_{\ell}$ casts the vote $\psi_{\ell}=\left(\operatorname{tag}_{\ell}, a_{\ell}, C_{\ell, j_{\ell}}^{\left(a_{\ell}\right)}\right)$. The EA receives the vote and updates its state st by appending $\psi_{\ell}$. The receipt $\alpha_{\ell}$ of $V_{\ell}$ is the vote $\psi_{\ell}$ and the part $s_{\ell}^{\left(1-a_{\ell}\right)}$ used for audit.

The Tally(Pub) protocol. Let $\tilde{\mathcal{V}}$ be the set of the voters that have voted successfully.

- For each $V_{\ell} \in \tilde{\mathcal{V}}$, the EA uses $\left(\operatorname{tag}_{\ell}, a_{\ell}\right)$ from $\psi_{\ell}$ to recover the respective audit information $s_{\ell}^{\left(1-a_{\ell}\right)}$ from $s_{\ell}$. Then, it sends to BB the list $\left\{\left(\psi_{\ell}, s_{\ell}^{\left(1-a_{\ell}\right)}\right)\right\}_{V_{\ell} \in \tilde{\mathcal{V}}}$. It also opens all the vote-code commitments, $\left\{U_{\ell, j}^{(a)}\right\}_{\ell \in[n], j \in[m]}^{a \in\{0,1\}}$, by sending the list of pairs $\left\{\left(C_{\ell, j}^{(a)}, t_{\ell, j}^{(a)}\right)\right\}_{\ell \in[n], j \in[m]}^{a \in\{0,1\}}$ to the BB. 
- The EA, for every $\psi_{\ell}$ corresponding to a $V_{\ell} \in \tilde{\mathcal{V}}$ :

(i). locates the decommitted vote-code $C_{\ell, j_{\ell}^{\prime}}^{\left(a_{\ell}\right)}$ that matches the cast votecode $C_{\ell, j_{\ell}}^{\left(a_{\ell}\right)}$. Then, it marks the vote-code $C_{\ell, j_{\ell}^{\prime}}^{\left(a_{\ell}\right)}$ as 'voted' and adds the corresponding commitment $E_{\ell, j_{\ell}^{\prime}}^{\left(a_{\ell}\right)}$ into the set $\mathbf{E}_{\text {tally }}$ (initially empty). Recall that $j_{\ell}^{\prime}=\pi_{\ell}^{\left(a_{\ell}\right)}\left(j_{\ell}\right)$.

(ii). adds all the commitments $\left\{E_{\ell, j}^{\left(1-a_{\ell}\right)}\right\}_{j \in[m]}$ that correspond to the votecodes in $s_{\ell}^{\left(1-a_{\ell}\right)}$ into the set $\mathbf{E}_{\text {open }}$ (initially empty).

When finalised, $\mathbf{E}_{\text {tally }}$ includes the collection of votes that will be counted (homomorphically) and $\mathbf{E}_{\text {open }}$ includes the information that will be used for verifying ballot correctness. After this happens, EA posts to the BB the list of marked vote-codes along with $\mathbf{E}_{\text {tally }}$ and $\mathbf{E}_{\text {open }}$.

- The EA produces and posts to the BB all the verifier's challenges $\left\{\rho_{E}\right\}_{E \in \mathbf{E}_{\text {tally }}}$ of the $\Sigma$-protocols for the validity of the commitments in $\mathbf{E}_{\text {tally }}$, as determined in Figure 3 (second move). The extraction of the challenges is done via the randomness contributed by the voters' coin-flips. The extraction method that is used is described in Section 3.4.

- The EA prepares and posts to the BB all the post-audit data $\left\{\phi_{2, E}\right\}_{E \in \mathbf{E}_{\text {tally }}}$ of the $\Sigma$-protocols for verifying the validity of the commitments in $\mathbf{E}_{\text {tally }}$, as determined in Figure 3 (third move). Thus, for each commitment in $\mathbf{E}_{\text {tally }}$ there is a triple of pre-audit data, challenge and post-audit data that form a complete $\Sigma$ proof of a valid commitment to some encoded candidate.

- EA performs homomorphic tally by computing $E_{\text {sum }}=\prod_{E \in \mathbf{E}_{\text {tally }}} E$ and preparing $(T, R)$ as the opening of $E_{\text {sum. }}$. The additive homomorphic property implies that $T$ is the election result encoded in the number system with base $N=n+1$ and it is committed under randomness $R$, which is the sum of all the randomness used for the commitments in $\mathbf{E}_{\text {tally }}$. Next, EA opens all the commitments in $\mathbf{E}_{\text {open }}$. Let Open be the set of these openings. Finally, it sends Open, $E_{\text {sum }}$ and $(T, R)$ to the BB.

- In the end of the process, BB contains the list of the marked vote-codes, as well as

$$
\begin{aligned}
& \text { Pub, }\left\{\left(C_{\ell, j}^{(a)}, t_{\ell, j}^{(a)}\right)\right\}_{\ell \in[n], j \in[m]}^{a \in\{0,1\}},\left(\mathbf{E}_{\text {tally }}, E_{\text {sum }},(T, R)\right), \\
& \text { (Open, } \left.\mathbf{E}_{\text {open }}\right),\left\{\rho_{E}\right\}_{E \in \mathbf{E}_{\text {tally }}},\left\{\phi_{2, E}\right\}_{E \in \mathbf{E}_{\text {tally }}}
\end{aligned}
$$

$\operatorname{Result}(\tau)$. The election result $R_{\tau}$ is derived by the following decoding algorithm:

$$
\begin{aligned}
& \text { Set } X \leftarrow T ; \\
& \text { For } j=1, \ldots, m: \\
& \text { - } x_{j} \leftarrow X \bmod (n+1) ; \\
& \text { - } X \leftarrow\left(X-x_{j}\right) /(n+1) ; \\
& \text { Return }\left\langle x_{1}, \ldots, x_{m}\right\rangle ;
\end{aligned}
$$

The correctness of the algorithm (and our system) is shown in Theorem 3. 
$\operatorname{Verify}(\tau, \alpha)$. Initially, $\alpha$ is parsed as $\left(\operatorname{tag}, a, C, s^{(1-a)}\right)$. The algorithm returns 1 only if the following checks are valid:

(i). All committed information in $\tau$ is associated with $n$ ballots indexed under different tags and no two vote-codes under the same tag are marked as 'voted'.

(ii). Let $\hat{C}$ be a vote-code that appears in part $\hat{s}^{(\hat{a})}$ of some ballot and has been marked as 'voted'. Then, only the committed information for the other part $\hat{s}^{(1-\hat{a})}$ of this ballot has been opened.

(iii). All the complete $\Sigma$ proofs that are associated with commitments in $\mathbf{E}_{\text {tally }}$ are valid.

(iv). $E_{\text {sum }}=\prod_{E \in \mathbf{E}_{\text {tally }}} E$.

(v). All the openings of the commitments are valid.

(vi). tag equals some $\operatorname{tag}_{\ell}$ in $\tau$ for some $\ell \in[n]$ and it holds that $a=a_{\ell}$.

(vii). The vote-code that is marked as 'voted' and is associated to $\operatorname{tag}_{\ell}$ is $C$ where $\ell$ is as in item (vi).

(viii). The correspondence of candidate encodings to vote-codes revealed in the opening of the commitments $\left\{\left(U_{\ell, j}^{\left(1-a_{\ell}\right)}, E_{\ell, j}^{\left(1-a_{\ell}\right)}\right)\right\}_{j \in[m]}$ where $\ell$ is as in item (vi), is equal to the one defined in $s^{(1-a)}$.

\subsection{Correctness of our e-voting system}

We prove the correctness of our system in the following theorem. In the remaining of the paper, we assume that $n \cdot(n+1)^{m-1}<q$.

Theorem 3. Let $q$ be the size of the group for the commitment scheme described in Section 3.1 and assume that $n \cdot(n+1)^{m-1}<q$. Then, the e-voting system described in Section 3.5 has perfect correctness.

Proof. See full version of the paper.

\subsection{Example of our e-Voting System}

For the better understanding of our e-voting system, we provide a toy example of a referendum where $P_{1}=$ YES, $P_{2}=\mathrm{NO}$ are the candidates and $\mathcal{V}$ consists of three voters $V_{1}, V_{2}, V_{3}$. Our goal is to familiarize the reader with the functionality of our system so, for simplicity, we deviate from the description in Section 3.5 by not including $\Sigma$-protocol proofs.

EA generates the vote-codes for the ballots $s_{1}, s_{2}$ and $s_{3}$ of $V_{1}, V_{2}$ and $V_{3}$ as

$$
\begin{aligned}
& \left(C_{1,1}^{(0)}=27935, C_{1,2}^{(0)}=75218, C_{1,1}^{(1)}=84439, C_{1,2}^{(1)}=77396\right), \\
& \left(C_{2,1}^{(0)}=58729, C_{2,2}^{(0)}=45343, C_{2,1}^{(1)}=14582, C_{2,2}^{(1)}=93484\right), \\
& \left(C_{3,1}^{(0)}=52658, C_{3,2}^{(0)}=65864, C_{3,1}^{(1)}=84373, C_{3,2}^{(1)}=49251\right)
\end{aligned}
$$

respectively. The double ballots $s_{1}, s_{2}, s_{3}$ are labelled by the tags 101,102, 103 respectively and are formed as follows: 


\begin{tabular}{|c|c|c|c|c|c|}
\hline \multicolumn{2}{|c|}{101} & \multicolumn{2}{|c|}{102} & \multicolumn{2}{|c|}{103} \\
\hline 27935 & YES & 58729 & YES & 52658 & YES \\
\hline 75218 & $\mathrm{NO}$ & 45343 & $\mathrm{NO}$ & 65864 & $\mathrm{NO}$ \\
\hline & YES & 14582 & $\mathrm{Y}$ & שו & $\mathrm{YES}$ \\
\hline 77396 & $\mathrm{NO}$ & 93484 & $\mathrm{NO}$ & 49251 & $\mathrm{NO}$ \\
\hline
\end{tabular}

EA prepares the commitments to each vote-code and the encoding of the candidate that they correspond. The commitment for YES (resp. NO) is a commitment to $(3+1)^{0}=1$ (resp. $\left.(3+1)^{1}=4\right)$. Next, it chooses whether the commitments of the vote-code and candidate pairs are going to be ordered in the $\mathrm{BB}$ as they are in the ballot part, or swapped. For example, assume that for the ballot $s_{1}$, EA chooses to leave the order in ballot part (0) intact and to swap the pairs in ballot part (1). Then, the information posted in the BB for $s_{1}$ would have the following form:

\begin{tabular}{|c|c|}
\hline \multicolumn{2}{|c|}{101} \\
\hline $\operatorname{Com}_{\mathrm{ck}}\left(27935 ; t_{1,1}^{(0)}\right)$ & $\operatorname{Com}_{\mathrm{ck}}\left(1 ; r_{1,1}^{(0)}\right)$ \\
$\operatorname{Com}_{\mathrm{ck}}\left(75218 ; t_{1,2}^{(0)}\right)$ & $\operatorname{Com}_{\mathrm{ck}}\left(4 ; r_{1,2}^{(0)}\right)$ \\
\hline $\operatorname{Com}_{\mathrm{ck}}\left(77396 ; t_{1,2}^{(1)}\right)$ & $\operatorname{Com}_{\mathrm{ck}}\left(4 ; r_{1,2}^{(1)}\right)$ \\
$\operatorname{Com}_{\mathrm{ck}}\left(84439 ; t_{1,1}^{(1)}\right)$ & $\operatorname{Com}_{\mathrm{ck}}\left(1 ; r_{1,1}^{(1)}\right)$ \\
\hline
\end{tabular}

Suppose that $V_{1}$ votes for NO using ballot part (1), $V_{2}$ votes for YES using ballot part (1) and $V_{3}$ votes for YES using ballot part (0). Then, the votes cast by $V_{1}, V_{2}$ and $V_{3}$ are $(101,1,77396),(102,1,14582)$ and $(103,0,52568)$ respectively. The receipts that the voters receive are

\begin{tabular}{|c|c|c|c|c|c|}
\hline$(101,1$ & 77396) & $(102$, & 14582) & $(103,0$ & 52568) \\
\hline 27935 & YES & 58729 & YES & 84373 & YES \\
\hline 75218 & $\mathrm{NO}$ & 45343 & $\mathrm{NO}$ & 49251 & $\mathrm{NO}$ \\
\hline
\end{tabular}

The coins that $V_{1}, V_{2}$ and $V_{3}$ have flipped, are $a_{1}=1, a_{2}=1$ and $a_{3}=0$ respectively. Hence, we get internal randomness, $(1,1,0)$, of 3 bits (which would be the "weak source" of randomness used for the extraction of the challenge of the $\Sigma$ protocols). After the voting ends, EA opens the vote-code commitments, marks the cast vote-codes 77396,14582 and 52658 and includes the corresponding encoded candidate commitments $\operatorname{Com}_{\mathrm{ck}}\left(4 ; r_{1,2}^{(1)}\right), \operatorname{Com}_{\mathrm{ck}}\left(1 ; r_{2,1}^{(1)}\right)$ and $\operatorname{Com}_{\mathrm{ck}}\left(1 ; r_{3,1}^{(0)}\right)$ in the tally set. Next, EA performs homomorphic tally, by computing the product of the above encoded candidate commitments as

$$
E_{\text {sum }}=\operatorname{Com}_{\mathrm{ck}}\left(4 ; r_{1,2}^{(1)}\right) \cdot \operatorname{Com}_{\mathrm{ck}}\left(1 ; r_{2,1}^{(1)}\right) \cdot \operatorname{Com}_{\mathrm{ck}}\left(1 ; r_{3,1}^{(0)}\right)=\operatorname{Com}_{\mathrm{ck}}\left(6 ; r_{1,2}^{(1)}+r_{2,1}^{(1)}+r_{3,1}^{(0)}\right) \text {. }
$$

Then, EA publishes $E_{\text {sum }}$, along with the opening of $E_{\text {sum }}$ at value $\left(6 ; r_{1,2}^{(1)}+\right.$ $\left.r_{2,1}^{(1)}+r_{3,1}^{(0)}\right)$. The result is derived by computing $x_{1}=6 \bmod 4=2$ and $x_{2}=$ 
$\left(\left(6-x_{1}\right) / 4\right) \bmod 4=1$, which is interpreted as two votes for YES and one for NO.

In the verification phase, the EA opens the commitments in the ballot parts that the voters selected for auditing. For example, $V_{1}$ would check the consistency of her receipt with audit information in the $\mathrm{BB}$, as illustrated below

\begin{tabular}{|c|c|c|c|}
\hline \multicolumn{3}{|c|}{101} \\
\hline 27935 & YES & $\left(1, r_{1,1}^{(0)}\right)$ & $\operatorname{Com}_{\mathrm{ck}}\left(1 ; r_{1,1}^{(0)}\right)$ \\
75218 & NO & $\left(4, r_{1,2}^{(0)}\right)$ & $\operatorname{Com}_{\mathrm{ck}}\left(4 ; r_{1,2}^{(0)}\right)$ \\
\hline 77396 & VOTED & & $\operatorname{Com}_{\mathrm{ck}}\left(4 ; r_{1,2}^{(1)}\right)$ \\
84439 & & & $\operatorname{Com}_{\mathrm{ck}}\left(1 ; r_{1,1}^{(1)}\right)$ \\
\hline
\end{tabular}

\begin{tabular}{|l|c|}
\hline \multicolumn{2}{|c|}{ Encodings } \\
\hline YES & 1 \\
NO & 4 \\
\hline
\end{tabular}

Observe that, as we will prove, the cut-and-choose verification that $V_{1}$ performs, does not reveal her vote even to a party that obtains her receipt. This is because the cast vote-code alone does not leak any information about the associated candidate, while the entirely opened auditing part only serves as a check that the correspondence of the vote-codes and candidates in this part has not been tampered with. Therefore, $V_{1}$ can delegate the task of verification to a third party, without compromising her privacy.

\section{Security of Our e-Voting System}

In this section, we prove the security of our system in the definitional framework presented in Sections 2.4 and 2.3.

\subsection{E2E Verifiability of Our e-Voting System}

We prove that our e-voting system achieves E2E verifiability information theoretically in the standard model. We follow the notation in Figure 1 and the description in 3.5 .

Theorem 4. Let $n$ be the number of all voters and $m$ be the number of candidates. Let $q$ be the size of the group for the commitment scheme described in Section 3.1. The e-voting system described in 3.5 achieves E2E verifiability information theoretically with error $\left.(1 / 2)^{d}+\epsilon(m, n,\lceil n /\lfloor\log q\rfloor\rceil, \theta-1)\right)$, where $\theta$ is the number of honest successful voters, $d$ is the tally deviation that the adversary wants to achieve and $\epsilon(m, n,\lceil n /\lfloor\log q\rfloor\rceil, \theta-1)$ is the soundness error of the $\Sigma$ protocol performed by the EA given in Theorem 2.

Proof. Without loss of generality (w.l.o.g.), we assume that in any adversarial execution as described in the E2E verifiability game $G_{\mathrm{E} 2 \mathrm{E}-\operatorname{Ver}}^{\mathcal{A}, \mathcal{E}, \theta}\left(1^{\lambda}, m, n\right)$, exactly $n$ ballots are tabulated on $\tau$ under $n$ different tags and all vote-codes marked as 'voted' correspond to different tags (if such deviations happen the transcript is immediately rejected). In the same spirit, we assume there is no double ballot 
that both parts have been opened and that all double ballots for honest voters in $\tilde{\mathcal{V}}$ are well-formed, otherwise they would not engage in the Cast protocol. Finally, we recall that the adversary cannot modify the history of the transcript since it does not have control over the BB. As a first step, we construct a vote extractor $\mathcal{E}$ for our system as follows:

Construction of the vote extractor. $\mathcal{E}$ has input $\tau$ and the set of receipts $\left\{\alpha_{\ell}\right\}_{V_{\ell} \in \tilde{\mathcal{V}}}$, where $\tilde{\mathcal{V}}$ is the set of the honest voters that voted successfully. Let $t \leq|\tilde{\mathcal{V}}|$ be the number of different tags that appear in $\left\{\alpha_{\ell}\right\}_{V_{\ell} \in \mathcal{V}}{ }^{6}$. If $\operatorname{Result}(\tau)=\perp$ (i.e., the transcript is not meaningful), then $\mathcal{E}$ outputs $\perp$. Otherwise, $\mathcal{E}$ (arbitrarily) arranges the voters in $\mathcal{V} \backslash \tilde{\mathcal{V}}$ and the tags not included in $\left\{\alpha_{\ell}\right\}_{V_{\ell} \in \tilde{\mathcal{V}}}$ as $\left\langle V_{\ell}^{\mathcal{E}}\right\rangle_{\ell \in[n-|\tilde{\mathcal{V}}|]}$ and $\left\langle\operatorname{tag}_{\ell}^{\mathcal{E}}\right\rangle_{\ell \in[n-t]}$ respectively. Next, for every $\ell \in[n-|\tilde{\mathcal{V}}|]$ :

1. If there is no marked as 'voted' vote-code that is associated with $\operatorname{tag}_{\ell}^{\mathcal{E}}$, then $\mathcal{E}$ sets $\mathcal{U}_{\ell}^{\mathcal{E}}=\emptyset$ (encoded as the zero vector) which is interpreted as an abort for voter $V_{\ell}^{\mathcal{E}}$.

2. If there is a 'voted' vote-code $C_{\ell, j}^{(a)}$ that is associated with $\operatorname{tag}_{\ell} \mathcal{E}$, then $\mathcal{E}$ brute-force opens the respective encoded candidate commitment $E_{\ell, j}^{(a)}$ to a value $\mathrm{Open}_{\ell}$ (recall the commitment is perfectly binding). If Open $\mathrm{O}_{\ell}$ is a valid encoding (i.e. Open $\left._{\ell} \in\left\{(n+1)^{0},(n+1)^{1}, \ldots,(n+1)^{m-1}\right\}\right)$ of a candidate $\mathcal{P}_{\ell}^{\mathcal{E}}$, then $\mathcal{E}$ sets $\mathcal{U}_{\ell}^{\mathcal{E}}=\left\{\mathcal{P}_{\ell}^{\mathcal{E}}\right\}$. Otherwise, it outputs $\perp$.

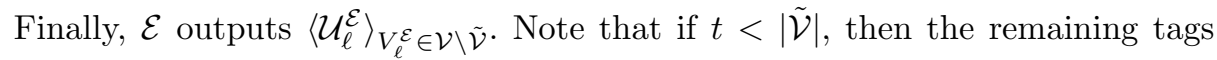
$\operatorname{tag}_{n-|\tilde{\mathcal{V}}|+1}^{\mathcal{E}}, \ldots, \operatorname{tag}_{n-t}^{\mathcal{E}}$ are ignored by $\mathcal{E}$.

Based on the above vote extractor, we will prove the E2E verifiability of our scheme. Assume an adversary $\mathcal{A}$ that wins the game $G_{\mathrm{E} 2 \mathrm{E}-\mathrm{E} \text {-Ver }}^{\mathcal{A}, \mathcal{E}, d}\left(1^{\lambda}, m, n\right)$. Namely, $\mathcal{A}$ breaks E2E verifiability by allowing at least $\theta$ honest successful voters and achieving tally deviation $d$. Since there is at least one honest voter that performs verification $(\theta>0)$, w.l.o.g. we assume that $\mathcal{A}$ always outputs meaningful transcripts.

Let $F$ be the event that there exists a committed value in $\tau$ which is marked to be counted and invalid (i.e., it is in $\mathbf{E}_{\text {tally }}$ but it is not a commitment to some candidate encoding). Since condition (i) of $G_{\mathrm{E} 2 \mathrm{E}-\mathrm{Ver}}^{\mathcal{A}, \mathcal{E}, d, \theta}\left(1^{\lambda}, m, n\right)$ holds, we have that there are at least $\theta$ honest voters. However, the soundness error of the $\Sigma$ - protocol is going to be affected by the fact that the invalid commitment is in a specific ballot part. The min entropy of all the coins given the fact that the adversary knows the coin of the invalid commitment in order to win, is at least the min entropy of all the coins minus 1 bit (i.e., the entropy of that bit). Therefore, by applying Theorem 2 for min entropy equal to $\theta-1$, we have that each $\Sigma$ protocol has soundness error $\epsilon(m, n,\lceil n /\lfloor\log q\rfloor\rceil, \theta-1)$. Hence, the probability that a committed value is invalid while verification accepts is no

\footnotetext{
${ }^{6}$ This implies that the ballot audit for all voters in $\tilde{\mathcal{V}}$ focuses on a list of $t$ tabulated ballots on the BB. Thus, an adversary may inject $|\tilde{\mathcal{V}}|-t$ ballots for candidate selections of its choice that will be counted in the final tally as if they were honest.
} 
more than $\epsilon(m, n,\lceil n /\lfloor\log q\rfloor\rceil, \theta-1)$. Since there is at least one honest voter that verifies, we conclude that

$$
\operatorname{Pr}\left[G_{\mathrm{E} 2 \mathrm{E}-\operatorname{E}, \boldsymbol{V} \text {-Vr }}^{\mathcal{A}, \boldsymbol{B}}\left(1^{\lambda}, m, n\right)=1 \wedge F\right] \leq \epsilon(m, n,\lceil n /\lfloor\log q\rfloor\rceil, \theta-1) .
$$

Assume that $F$ does not occur. Thus, all marked committed values in $\mathbf{E}_{\text {tally }}$ correspond to a valid candidate encoding. This implies that (a) the maximum deviation per marked commitment that $\mathcal{A}$ may achieve is 1 (the vote is counted for a candidate other than the intended one) and (b) $\mathcal{E}$ does not output $\perp$ (it returns a vector $\left\langle\mathcal{U}_{\ell}^{\mathcal{E}}\right\rangle_{V_{\ell}^{\mathcal{E}} \in \mathcal{V} \backslash \tilde{\mathcal{V}}}$ ), so $\mathcal{A}$ wins because (i),(ii) and (iii-a) hold. The auditor can verify that $E_{\text {sum }}$ is equal to the homomorphic commitment $\prod_{E \in \mathbf{E}_{\text {tally }}} E$. Due to the perfect binding of the commitment scheme, the tally $f\left(\left\langle\mathcal{U}_{\ell}^{\mathcal{E}}\right\rangle_{V_{\ell}^{\mathcal{E}} \in \mathcal{V} \backslash \tilde{\mathcal{V}}}\right)$ that $\mathcal{E}$ estimates as non-honest votes, is correctly included in the adversarial result that derives from the opening $(T, R)$ of $E_{\text {sum }}$. Thus, the deviation from the intended result that $\mathcal{A}$ achieves, derives only by miscounting the honest votes. This may be achieved by $\mathcal{A}$ in two different possible ways:

1. Modification attacks: modify the committed information as compared with the one in an honest voter's ballot (e.g., alter the vote-code and candidate correspondence). The deviation achieved by this type of attack is at most 1.

2. Clash attacks: instruct $r$ honest voters whose ballots are indexed under the same tag to vote so that the votes of any $r-1$ out of these $r$ voters are all different than some fixed $r-1$ committed votes that are ignored by $\mathcal{E}$ (either cast by corrupted voters or initially injected in $\tau$ by $\mathcal{A}$ ). All $r$ voters verify the correct counting of their votes by auditing the same information on the $\mathrm{BB}$ and hence miss the injected votes that produce the tally deviation. The deviation achieved by this type of attack is $r-1$.

In the case where all ballot information is committed consistently on the BB without being deleted or replaced, the adversary can only perform a combination of these two attacks on the honest voters. Indeed, if all honestly cast votes are in one-to-one correspondence with the correct encoded candidate commitments, then the perfect binding property ensures that the opening of the homomorphic tally matches the intended result.

Let $\tilde{\mathcal{V}}_{1}, \ldots, \tilde{\mathcal{V}}_{t}$ be the partition of $\tilde{\mathcal{V}}$ s.t. each of these subsets consists of honest voters that their receipts (hence their ballots) are indexed under the same tag. These subsets are created adaptively, according to the strategy of $\mathcal{A}$, under the constraint that $|\tilde{\mathcal{V}}| \geq \theta$. Note that there are $|\tilde{\mathcal{V}}|-t$ ignored tags in vote extraction, while $\sum_{i \in[t]}\left(\left|\tilde{\mathcal{V}}_{i}\right|-1\right)=|\tilde{\mathcal{V}}|-t$. This implies that the adversary can perform clash attacks in all these subsets, with maximum possible deviation. We will prove that given that $F$ does not occur, the success probability of $\mathcal{A}$ is no more than $(1 / 2)^{d}$, whatever its strategy might be.

We observe that in order for $\mathcal{A}$ to win, all voters in $\mathcal{V}_{i}$ must have the same receipt, or else inconsistencies will cause verification to fail. To achieve this, $\mathcal{A}$ must instruct the voters from the same subset to vote so that they all cast the same vote-code (otherwise two marked vote-codes under the same tag should 
appear) and create the corresponding audit ballot part identically for each auditing voter. In detail, in order for $\mathcal{A}$ to win, the following must hold for each $\tilde{\mathcal{V}}_{i}, i \in[t]:$

1. There is a representative vote-code $C_{i}$ that appears in part $(a)$ of all the double ballots of the voters in $\tilde{\mathcal{V}}_{i}$. The voters must select this part to vote by casting $C_{i}$. Therefore, the coin-flippings of the auditing voters must be consistent, in the sense that they correspond to ballot parts that contain a consistent vote-code. There can be at most 2 consistent coin-flips (i.e., either all coins are flipped to 0 or all coins are flipped to 1 ). Thus, the probability of consistent coin-flipping in $\tilde{\mathcal{V}}_{i}$ is at most $2 / 2^{|\tilde{\mathcal{V}}|_{i}}=(1 / 2)^{|\tilde{\mathcal{V}}|_{i}-1}$. In addition, the ballot parts that will be used for auditing must contain the same information, up to a permutation of the vote-code and candidate pairs.

2. If $\mathcal{A}$ wants to achieve $\left|\tilde{\mathcal{V}}_{i}\right|$ deviation exploiting the voters in $\mathcal{V}_{i}$, then it must perform a modification attack in at least one voter $V$ in $\tilde{\mathcal{V}}_{i}$. This is because if all voters' ballots are consistent to the corresponding committed information in $\tau$, then by performing only a clash attack in $\tilde{\mathcal{V}}_{i}, \mathcal{A}$ can achieve deviation by at most $\left|\tilde{\mathcal{V}}_{i}\right|-1$, as described above. However, the modification comes with a loss of $1 / 2$ success probability, since $\mathcal{A}$ must also guess which is the part that $V$ is going to use for voting. Indeed, if $V$ chooses to audit the modified part of the ballot, then she will detect the attack. Therefore, all voters in $\mathcal{V}_{i}$ must perform a consistent coin-flip that agrees with the coinflip of $V$. It is straightforward that in case of a single modification attack this event happens with $1 / 2 \cdot(1 / 2)^{\left|\tilde{\mathcal{V}}_{i}\right|-1}=(1 / 2)^{\left|\tilde{\mathcal{V}}_{i}\right|}$ probability. Moreover, in case $\tilde{\mathcal{V}}_{i} \geq 2$, performing two modification attacks does not lead to any improvement in terms of probability or maximum deviation.

We note that the above arguments hold trivially, if $\tilde{\mathcal{V}}_{i}$ is a singleton. Let $\mathbf{X}$ be the set of subsets from $\left\{\tilde{\mathcal{V}}_{1}, \ldots, \tilde{\mathcal{V}}_{t}\right\}$ that $\mathcal{A}$ performs clash attacks and $\mathbf{Y}$ the collection that $\mathcal{A}$ performs a modification attack on at least one voter in each of the subsets. According to the previous arguments, we have the following cases: (i) for each $\mathcal{V}_{i} \in \mathbf{X} \backslash \mathbf{Y}$ the maximum deviation is $\left|\tilde{\mathcal{V}}_{i}\right|-1$, (ii) for each $\mathcal{V}_{i} \in \mathbf{Y} \backslash \mathbf{X}$ the maximum deviation is 1 , (iii) for each $\mathcal{V}_{i} \in \mathbf{X} \cap \mathbf{Y}$ the maximum deviation is $\left|\tilde{\mathcal{V}}_{i}\right|$ and (iv) for each $\mathcal{V}_{i} \in\left\{\tilde{\mathcal{V}}_{1}, \ldots, \tilde{\mathcal{V}}_{t}\right\} \backslash(\mathbf{X} \cup \mathbf{Y})$ the maximum deviation is 0 . For brevity, let $x=|\mathbf{X}|$ and $y=|\mathbf{Y}|$. Therefore, we have that the tally deviation from the intended result that $\mathcal{A}$ achieves is at most

$$
\sum_{\mathcal{V}_{i} \in \mathbf{X} \backslash \mathbf{Y}}\left(\left|\tilde{\mathcal{V}}_{i}\right|-1\right)+\sum_{\mathcal{V}_{i} \in \mathbf{Y} \backslash \mathbf{X}} 1+\sum_{\mathcal{V}_{i} \in \mathbf{X} \cap \mathbf{Y}}\left|\tilde{\mathcal{V}}_{i}\right|=\sum_{\mathcal{V}_{i} \in \mathbf{X}}\left|\tilde{\mathcal{V}}_{i}\right|-x+y \leq|\tilde{\mathcal{V}}|-x+y
$$

We will now upper bound the success probability of $\mathcal{A}$. Since $\left\{\tilde{\mathcal{V}}_{1}, \ldots, \tilde{\mathcal{V}}_{t}\right\}$ is a partition of $\tilde{\mathcal{V}}$, we have that $\mathcal{A}$ must not be detected by all the voters in all of 
these subsets. So,

$$
\begin{aligned}
\operatorname{Pr}\left[G_{\mathrm{E} 2 \mathrm{E}-\operatorname{Ver}}^{\mathcal{A}, \mathcal{E}, \theta}\left(1^{\lambda}, m, n\right)=1 \mid \neg F\right] & \leq \prod_{\mathcal{V}_{i} \in \mathbf{Y}}(1 / 2)^{\left|\tilde{\mathcal{V}}_{i}\right|} \cdot \prod_{\mathcal{V}_{i} \in\left\{\tilde{\mathcal{V}}_{1}, \ldots, \tilde{\mathcal{V}}_{t}\right\} \backslash \mathbf{Y}}(1 / 2)^{\left|\tilde{\mathcal{V}}_{i}\right|-1}= \\
& =(1 / 2)^{\sum_{\mathcal{V}_{i} \in \mathbf{Y}}\left|\tilde{\mathcal{V}}_{i}\right|+\sum_{\mathcal{V}_{i} \in\left\{\tilde{\mathcal{V}}_{1}, \ldots, \tilde{\mathcal{V}}_{t}\right\} \backslash \mathbf{Y}}\left(\left|\tilde{\mathcal{V}}_{i}\right|-1\right)}= \\
& =(1 / 2)^{|\tilde{\mathcal{V}}|-(t-y)} \leq(1 / 2)^{|\tilde{\mathcal{V}}|-x+y},
\end{aligned}
$$

because $x \leq t$. In order for $\mathcal{A}$ to win, it must hold that $|\tilde{\mathcal{V}}|-x+y \geq d$ (condition (iii-a) holds), therefore

$$
\operatorname{Pr}\left[G_{\mathrm{E} 2 \mathrm{E}-\operatorname{Ver}}^{\mathcal{A}, \mathcal{V}, \theta}\left(1^{\lambda}, m, n\right)=1 \wedge \neg F\right] \leq \operatorname{Pr}\left[G_{\mathrm{E} 2 \mathrm{E}-\operatorname{Ver}}^{\mathcal{A}, \mathcal{E}, d,}\left(1^{\lambda}, m, n\right)=1 \mid \neg F\right] \leq(1 / 2)^{d} .
$$

By adding (1),(2) we conclude that

$$
\operatorname{Pr}\left[G_{\mathrm{E} 2 \mathrm{E}-\text { Ver }}^{\mathcal{A}, \mathcal{E}, \theta}\left(1^{\lambda}, m, n\right)=1\right] \leq(1 / 2)^{d}+\epsilon(m, n,\lceil n /\lfloor\log q\rfloor\rceil, \theta-1) .
$$

Remark 1. Note that if the number of honest voters satisfies the bound $\theta=$ $\Omega(n \log \log m / \log q+\lambda)$, then the overall soundness error of the repeated $\Sigma$ protocol will be sufficiently small. For instance, in an election where there are $n=1000$ voters and $m=40$ candidates we can use a group with at least 500 bit prime order $q$. Assuming a number of $\theta=50$ honest voters ( $5 \%$ of total) we can divide the 1000 voter's coins into two challenges with 500 bits each (i.e. $k=2$ ). With these parameters the above theorem will have a verifiability error that is at most $2^{-43}+(1 / 2)^{d}$ where $d$ is the tally deviation. We remark that in this setting no deterministic extractor would be able to provide sufficient entropy and hence our ZK amplification technique is crucial.

\subsection{Voter Privacy/Receipt Freeness of Our e-Voting System}

In order to show our scheme satisfies privacy, we utilize complexity leveraging. Specifically, the system security parameter is configured such that breaking the hiding property of the underlying commitment scheme is much harder than guessing the challenge of the $\Sigma$ protocol; therefore, we can simulate the protocol's view by guessing the proof challenges without breaking the hiding property of the commitment scheme. Due to this proof technique, the number of corrupted voters $t$ should be polynomially related to the security parameter $\lambda$ in a certain way; while the total number of voters $n$ can be any function that is poly $(\lambda)$ (as long as the correctness requirement is fulfilled, cf. theorem 3). We emphasize that given a specific $n$, our system can support privacy for any desired number of adversarial voters $t<n$ (as long as a suitably large security parameter $\lambda$ is used).

Theorem 5. Assume there exists a constant $c, 0<c<1$ such that for any $2^{\lambda^{c}}$-time adversary $\mathcal{A}$, the advantage of breaking the hiding property of the commitment scheme is $\operatorname{Adv}_{\text {hide }}(\mathcal{A})=\operatorname{negl}(\lambda)$. Let $t=\lambda^{c^{\prime}}$ for some constant $c^{\prime}<c$. For any constant $m \in \mathbb{N}$ and $n=$ poly $(\lambda)$, the e-voting system described in Section 3.5 achieves voter privacy/receipt-freeness for at most $t$ corrupted voters. 
Proof. See full version of the paper.

\section{References}

1. Ben Adida. Helios: Web-based open-audit voting. In USENIX Security, 2008.

2. B. Barak, G. Kindler, R. Shaltiel, B. Sudakov, and A. Wigderson. Simulating independence: New constructions of condensers, ramsey graphs, dispersers, and extractors. J. ACM, 57(4):20:1-20:52, May 2010.

3. Carsten Baum, Ivan Damgård, and Claudio Orlandi. Publicly auditable secure multi-party computation. In SCN 2014 Proceedings, 2014.

4. Donald Beaver. Plug and play encryption. In CRYPTO'97, pages 75-89, 1997.

5. Josh Benaloh. Simple verifiable elections. USENIX, 2006.

6. Josh Cohen Benaloh and Dwight Tuinstra. Receipt-free secret-ballot elections (extended abstract). In STOC, 1994.

7. Josh Cohen Benaloh and Moti Yung. Distributing the power of a government to enhance the privacy of voters (extended abstract). In PODC, 1986.

8. David Bernhard, Véronique Cortier, Olivier Pereira, Ben Smyth, and Bogdan Warinschi. Adapting helios for provable ballot privacy. In ESORICS, 2011.

9. David Bernhard, Olivier Pereira, and Bogdan Warinschi. How not to prove yourself: Pitfalls of the Fiat-Shamir heuristic and applications to Helios. In Asiacrypt, 2012.

10. Manuel Blum, Paul Feldman, and Silvio Micali. Non-interactive zero-knowledge and its applications (extended abstract). In STOC, 1988.

11. David Chaum. Untraceable electronic mail, return addresses, and digital pseudonyms. Commun. ACM, 24(2):84-88, 1981.

12. David Chaum. Surevote: Technical overview. In Proceedings of the Workshop on Trustworthy Elections, WOTE, 2001.

13. David Chaum. Secret-ballot receipts: True voter-verifiable elections. IEEE Security E Privacy, 2(1):38-47, 2004.

14. David Chaum, Richard Carback, Jeremy Clark, Aleksander Essex, Stefan Popoveniuc, Ronald L. Rivest, Peter Y. A. Ryan, Emily Shen, Alan T. Sherman, and Poorvi L. Vora. Scantegrity II: end-to-end verifiability by voters of optical scan elections through confirmation codes. IEEE TIFS, 4(4):611-627, 2009.

15. Benoît Chevallier-Mames, Pierre-Alain Fouque, David Pointcheval, Julien Stern, and Jacques Traoré. On some incompatible properties of voting schemes. In Towards Trustworthy Elections, 2010.

16. Michael R. Clarkson, Stephen Chong, and Andrew C. Myers. Civitas: Toward a secure voting system. In IEEE Symposium on Security and Privacy, 2008.

17. Josh D. Cohen and Michael J. Fischer. A robust and verifiable cryptographically secure election scheme (extended abstract). In FOCS, 1985.

18. Josh D. Cohen and Michael J. Fischer. A robust and verifiable cryptographically secure election scheme (extended abstract). In FOCS, 1985.

19. United States Election Assistance Commission. Voluntary voting systems guidelines, 2005.

20. Ronald Cramer, Ivan Damgård, and Berry Schoenmakers. Proofs of partial knowledge and simplified design of witness hiding protocols. In CRYPTO, 1994.

21. Ronald Cramer, Matthew K. Franklin, Berry Schoenmakers, and Moti Yung. Multi-autority secret-ballot elections with linear work. In EUROCRYPT, 1996.

22. Ronald Cramer, Rosario Gennaro, and Berry Schoenmakers. A secure and optimally efficient multi-authority election scheme. ETT, 8(5):481-490, 1997. 
23. Olivier de Marneffe, Olivier Pereira, and Jean-Jacques Quisquater. Simulationbased analysis of E2E voting systems. In Frontiers of Electronic Voting, 2007.

24. Stéphanie Delaune, Steve Kremer, and Mark Ryan. Verifying privacy-type properties of electronic voting protocols. J. of Computer Security, 17(4):435-487, 2009.

25. Danny Dolev, Michael J Fischer, Rob Fowler T, Nancy A Lynch, and H. Raymond Strong. An efficient algorithm for byzantine agreement without authentication. Information and Control, 52:257-274, 1982.

26. Juan A. Garay, Aggelos Kiayias, and Nikos Leonardos. The bitcoin backbone protocol: Analysis and applications. In EUROCRYPT'15. Springer, 2015.

27. Jens Groth. Evaluating security of voting schemes in the universal composability framework. In $A C N S^{\prime} 04$, pages 46-60, 2004.

28. Eitan M. Gurari. Introduction to the theory of computation. Computer Science Press, 1989.

29. Ari Juels, Dario Catalano, and Markus Jakobsson. Coercion-resistant electronic elections. IACR Cryptology ePrint Archive, 2002:165, 2002.

30. Jesse Kamp and David Zuckerman. Deterministic extractors for bit-fixing sources and exposure-resilient cryptography. SIAM J. Comput., 36(5):1231-1247, 2006.

31. Steve Kremer, Mark Ryan, and Ben Smyth. Election verifiability in electronic voting protocols. In ESORICS, pages 389-404, 2010.

32. Ralf Küsters, Tomasz Truderung, and Andreas Vogt. Accountability: Definition and relationship to verifiability. IACR Cryptology ePrint Archive, 2010:236, 2010.

33. Ralf Küsters, Tomasz Truderung, and Andreas Vogt. A game-based definition of coercion-resistance and its applications. In CSF, pages 122-136, 2010.

34. Ralf Küsters, Tomasz Truderung, and Andreas Vogt. Verifiability, privacy, and coercion-resistance: New insights from a case study. In IEEE Symposium on Security and Privacy, pages 538-553. IEEE Computer Society, 2011.

35. David Lichtenstein, Nathan Linial, and Michael E. Saks. Imperfect random sources and discrete controlled processes. In STOC, pages 169-177, 1987.

36. Silvio Micali, Rafael Pass, and Alon Rosen. Input-indistinguishable computation. In FOCS, pages 367-378. IEEE Computer Society, 2006.

37. Tal Moran and Moni Naor. Receipt-free universally-verifiable voting with everlasting privacy. In CRYPTO, pages 373-392, 2006.

38. C. Andrew Neff. Practical high certainty intent verification for encrypted votes. Votehere, Inc. whitepaper, 2004.

39. Stefan Popoveniuc, John Kelsey, Andrew Regenscheid, and Poorvi Voral. Performance requirements for end-to-end verifiable elections. EVT/WOTE, 2010.

40. Ran Raz. Extractors with weak random seeds. STOC, 2005.

41. Kazue Sako and Joe Kilian. Receipt-free mix-type voting scheme - a practical solution to the implementation of a voting booth. In EUROCRYPT, 1995.

42. Jacob T. Schwartz. Fast probabilistic algorithms for verification of polynomial identities. J. $A C M, 27(4): 701-717,1980$.

43. Dominique Unruh and Jörn Müller-Quade. Universally composable incoercibility. IACR Cryptology ePrint Archive, 2009:520, 2009.

44. Filip Zagórski, Richard Carback, David Chaum, Jeremy Clark, Aleksander Essex, and Poorvi L. Vora. Remotegrity: Design and use of an end-to-end verifiable remote voting system. In $A C N S, 2013$.

45. Richard Zippel. Probabilistic algorithms for sparse polynomials. In EUROSAM, 1979. 